\title{
Abstracts of 30th Year Annual Meeting of Turkish Society of Neuroradiology with International Participation
}

Published online: 6 February 2021

(C) Springer-Verlag GmbH Germany, part of Springer Nature 2021

\section{5-CAN PERFUSION CHANGES EXPLAIN NEUROLOGIC SYMPTOMS IN SUBDURAL HEMATOMA}

$\underline{\text { Zakir Sakcl }}{ }^{1}$, Mehmet Gencturk ${ }^{2}$

Ümraniye Training and Research Hospital, Department of Radiology Radiology Istanbul-Turkey ${ }^{1}$ University of Minesota, Department of Radiology Radiology Mineapolis-United States ${ }^{2}$

\begin{abstract}
Introduction: Chronic subdural hematoma is not an uncommon disorder in elderly1. Although intensively investigated, numerous aspects, including the pathophysiology of clinical symptoms remain unclear2-4. Perfusion deficits may induce the transient neurologic symptoms seen in chronic subdural hematoma (cSDH). The aim of the present study was to quantify cerebral perfusion impairment in $\mathrm{CSDH}$.
\end{abstract}

Materials and Methods: We retrospectively evaluated the CT perfusion (CTP) images of 15 patients with SDH who were scanned for the clinical suspicion of acute infarct. Hematoma volume was measured using Vitrea software (Vital Images, division of Toshiba Medical, Minnetonka, MN), "3D tumor volume technique" in which radiologist selects the target density and software elects adjacent, contagious densities .CTP parameters of the cortex underlying the hematoma was compared with unaffected normal cortex on the contralateral side in unilateral SDH and unaffected cortex anterior or posterior to SDH in bilateral SDH. Correlation between the hematoma volume and CTP parameters were statistically searched.

Results: Mean hematoma volume was $45.3+/ 32.4 \mathrm{ml}$. The cerebral blood volume (CBV) and cerebral blood flow (CBF) were not significantly increased in the area under the hematoma (AUH) compared to the corresponding unaffected normal cortex (UNC). The mean CBV was $3.05 /+1.29$ in AUH and $2.83 /+0.96$ in UNC $(\mathrm{P}=0.097)$. The mean $\mathrm{CBF}$ was $32.89 /+$ 14.79 in $\mathrm{AUH}$ and $29.47 /+10.58$ in $\mathrm{UNC}(\mathrm{P}=0.11)$. The mean transit time was not significantly elevated in AUH compared to the NUC ( $6.07 /+1.05 \mathrm{vs}$ $5.85 /+0.85, \mathrm{p}=0.12$ ). Time to peak (TTP) was almost equal between these areas $(17.58 /+6.88$ vs $17.38 /+5.59, \mathrm{p}=0.82)$. In addition, no statistical significant correlation was found between hematoma volume and any of the global or hemispheric cerebral perfusion parameters.

Conclusion: Our study finds no evidence that neurologic symptoms associated with cSDH are due to changes in perfusion. There was no significant difference between perfusion parameters adjacent to and contralateral to cSDH. The study is limited by sample size. Also, as we compared perfusion between hemispheres in the same subjects, we could not determing if cSDH produced global rather than regional perfusion changes.

\section{4-DIAGNOSTIC VALUE OF HEMOSEQUENCE IN CRANIAL IMAGING \\ Nurdan Gocgun ${ }^{\text {I }}$ \\ Haseki Research And Training Hospital Radiology İstanbul-Turkey ${ }^{1}$}

Hemosequence is a sequence that helps standard magnetic resonance imaging (MRI) sequences separating blood products such as deoxyhemoglobin, methemoglobin, hemosiderin, calcium, metallic bodies, fat, and air intensities in the tissue. Although it is valuable especially in cerebrovascular diseases such as intracranial hemorrhage, it can also be helpful in more rare diagnoses. We aimed to present our case series in which we strengthen the diagnosis with the help of hemosequence. Clinical and radiological features of 47 cases were analyzed retrospectively. The cases were classified into three groups according to their etiological reasons; 1 . vascular 2. mass 3 .other reasons. 35 of the cases were due to vascular reasons, 5 were in the mass, and 7 were in the "other" group. Vascular lesions included hypertensive and amyloid microangiopathy, superficial siderosis, hemorrhagic infarction, sinus or cortical vein thrombosis, and vascular malformation. Two of the masses were hemorrhagic metastases, others were calcific meningioma, oligoastrocytoma, hemangioblastoma. In the 'Other' group, there was an infection, stroke mimicker, leukodystrophy, pituitary apoplexy, FAHR syndrome, and metabolic substance accumulation. Since the majority of microhemorrhages in hemosequence in ischemic stroke may predict secondary major hemorrhage that may occur with anticoagulant and or antiaggregant treatment; It is also important in terms of the treatment decision. Observation of bleeding in hemorrhagic metastases and hemangioblastoma and calcification in oligodendroglioma in our cases supported the diagnosis. Detection of hyperdense lesions on Computed Tomography (CT) in patients presenting with a clinical picture mimicking stroke made the distinction between sequela-calcification and hemorrhage difficult, but adding hemosequence to standard sequences in MRI helped in the differential diagnosis. In our case with leukodystrophy, the normal hemosequence excluded other metabolic causes with substance accumulation. In this case series, we wanted to emphasize the importance of hemosequence in the differential diagnosis in nonvascular central nervous system diseases as well as its known value in vascular diseases.

\section{6-IMAGING FINDINGS OF LATE PHASE BRAIN DEATH Oğuzhan Yıldız ${ }^{1}$, Şafak Parlak ${ }^{1}$, Gökçen Çoban Çifçi ${ }^{1}$ Hacettepe University School of Medicine Radiology Ankara-Turkey ${ }^{{ }^{\prime}}$}

Purpose: Brain death (BD) is a well-described entity with its clinical findings, confirmatory tests, and imaging features. Radiography may help diagnose $\mathrm{BD}$, especially when clinical assessment is inadequate, or the supportive tests are impossible. Although the radiologists are quite familiar with imaging findings in the early phase, the late phase findings are unknown. To our knowledge, there is no report addressing the magnetic resonance imaging (MRI) findings of the late phase of BD in the literature. In this paper, we present and highlight MRI findings of the late phase of $\mathrm{BD}$ in a patient with diffuse midline glioma (DMG). 
Presentation: A 7 year-old boy who had been diagnosed with DMG and received chemo-radiation therapy was taken home care with the family request. After 8 months, the family admitted to the hospital claiming the signs of healing in the patients' condition. Control MRI revealed dilated ventricles, increased T2 hyperintensity in white matter with cortical thinning, basal ganglia atrophy and pachymeningeal enhancement with diffuse dural sinus thrombosis. Diffusion-weighted MRI revealed severe centrifugal necrosis in the brain parenchyma. Perfusion-weighted MRI revealed the absence of perfusion in the brain parenchyma even in vanishing $\mathrm{DMG}$.

Conclusion: Imaging findings of the late phase of brain death is unknown. Conventional and advanced MRI findings of the long-term cerebral anoxia of a patient with DMG is our contribution to the literature.

1017-RISK FACTORS FOR EXTRASINUSOIDAL EXTENSION IN ACUTE INVASIVE FUNGAL SINUSITIS

Baris Korkmaz ${ }^{1}$, Rifat Ozpar ${ }^{1}$, Müfit Parlak ${ }^{1}$

Bursa Uludag University Radiology Bursa-Turkey ${ }^{1}$

Background and Purpose: Acute invasive fungal sinusitis (AIFS) is an aggressive sinonasal pathology with high morbidity and mortality rates. Extra-sinusoidal extension (ESE) is one of the main determiners for mortality and morbidity. Periantral infiltration (PI) may be an early sign of AIFS. In this study, we investigated the factors associated with ESE and PI of the AIFS, and we also examined the other radiological findings of AIFS.

Methods: 62 patients with pathologically and microbiologically confirmed AIFS between January 2012 and February 2020 were included in this study. Clinical and radiological findings were evaluated. To investigate the diagnostic performance of computer tomography (CT) about ESE and PI; magnetic resonance imaging (MRI) was accepted as the gold standard.

Results: ESE involvement was associated with several factors such as PI (OR:10.71, $\mathrm{p}=0.005, \% 95 \mathrm{CI}: 2.02-56.74)$, maxillary sinus infiltration (MSI) (OR:5.42, p=0.012, \%95 CI:1.45-20.24), zygomycetes class fungal pathogens (OR: 21.99, p=0.025, \%95 CI:1.48-325.71) and neutrophilia (14.05, $\mathrm{p}=0.014, \% 95 \mathrm{CI}: 1.71-115.4)$. Moreover, there were significant associations between bone destruction and PI $(\mathrm{p}=0.005)$ and between comorbidity and pathogen $(\mathrm{p}=0.002)$. The PI detection sensitivity of CT was $76.9 \%$ and specificity was $100 \%$. The ESE detection sensitivity of CT was $52.2 \%$ and specificity was $100 \%$. There was a low level of compatibility between CT and MR in ESE detection (\%51.5, $\mathrm{k}=0.264$, $\mathrm{p}=0.010$ )

Conclusion: Several clinical characteristics and imaging factors play a key role in predicting ESE of AIFS. Our results suggest that a physician should be particularly suspicious about ESE in the case of PI, MSI, zygomycetes class fungal pathogens and neutrophilia. The role of CT to detect PI and ESE is limited. Therefore, in such cases, an MRI scan may significantly improve the prognosis by obtaining an early diagnosis.

\section{2-EVALUATION OF ANATOMICAL VARIATIONS OF CRISTA GALLI ON PARANASAL CT}

VEYSEL ATILLA AYYILDIZ ${ }^{1}$, KENAN ÖZTÜRK ${ }^{2}$

Suleyman Demirel University Faculty of Medicine Department of Radiology ISPARTA-Turkey ${ }^{1}$ Suleyman Demirel University Faculty of Medicine Department of Anatomy ISPARTA-Turkey ${ }^{2}$

Introduction and purpose: Crista galli (CG) is located in the center of the cribriform plate of the ethmoid bone and is an important structure for neurosurgical procedures for tumors located anterior to the skull base (1). Knowing the location of the CG relative to the cribriform plate and the presence of pneumatization helps to prevent complications during surgical intervention. Therefore, we aimed to investigate the position of CG relative to lamina cribrosa and the presence of pneumatization.
Materials and methods: Our study was conducted on paranasal sinus computed tomography (CT) images of 70 patients ( 35 males, 35 females) aged between 20 and 84 years (mean 50.01 17.24 ), who were admitted to our hospital with a pre-diagnosis of acute sinusitis between May 2019 and May 2020. Images were obtained with a CT device (Siemens SOMATOM Definition AS 128-slice CT) with a slice thickness of 1 $\mathrm{mm}$, using parenchymal and bone filters at a dose of $80 \mathrm{kV}, 120 \mathrm{mAs}$, without contrast material in the axial and coronal planes. In addition, reformatted sagittal images were created and evaluated together with the images in other plans. The position of CG is divided into 3 types according to the classification of Hajiioannou (2). Type 1: CG's base is at the cribriform plate level. Type 2: Less than $50 \%$ of the height of the $\mathrm{CG}$ is below the level of cribriform plate. Type 3: More than $50 \%$ of the height of the CG is below the level of cribriform plate.

The ratio of pneumatized crista galli (PCG) was determined. PCGs are divided into three as type $\mathrm{S}$ (small), type $\mathrm{M}$ (moderate) and type L (large) according to the classification of Mladina et al. (1).

Results: According to the position of CG; 32 (45.7\%) patients were type $1,38(54.3 \%)$ patients were type 2 . However, type 3 could not be detected in any of the patients. PCG was observed in only 4 patients (5.7\%). Two $(2.9 \%)$ of the PCGs were type S, $1(1.4 \%)$ was type M and 1 was $(1.4 \%)$ type L. It was observed that 3 of the PCGs were connected to the frontal sinus, and one with the ethmoid sinus.

Discussion and conclusion: Studies on $\mathrm{CG}$ have been focused especially in the last 10 years (1-4). It is important to know the anatomy and pneumatization during surgical interventions for skull base tumors (2). Hajiioannou et al. (2) found $28.3 \%$ type 1, 63.6\% type 2 and $8.1 \%$ type $3 \mathrm{CG}$ and $14.1 \%$ PCG. Mladina et al. (1) reported $45.58 \%$ type 1 and $54.42 \%$ type 2 , but never type 3 . Our results regarding the position of $C G$ are quite similar to those of Mladina et al. (1).

$\mathrm{CG}$ is rarely pneumatized. The incidence of pneumatization has been reported to be $3-37.5 \%$ (1). PCG may be associated with the frontal sinus and anterior ethmoid cells. If there is such a connection, sinusitis in other connected sinuses has the potential to spread to the pneumatized part of the CG (2). Hajiioannou et al. (2) reported pneumatization in 5\% of type $2 \mathrm{CG}$ and $75 \%$ of type 3 CG. In our study, $25 \%$ of PCGs were observed in type 1, and $75 \%$ in type 2 CG. Based on these findings, Hajiioannou et al. (2) reported that pneumatization may be associated with CG descending into the nasal cavity. Classification and pneumatization of CG on paranasal sinus CT images; It enables to predict the complications that may arise during surgical procedures involving the nasal cavity, lamina cribrosa and CG, and to eliminate the complications that may occur in the early period.

\section{8-ADC VALUES AND RATIOS IN DIFFERENTIATION OF SCHWANNOMAS FROM MENINGIOMAS}

MERVE YAZOL $^{1}{ }^{1}$, HALIL OZER ${ }^{1}$, ALI YUSUF ONER ${ }^{1}$

Gazi University Faculty of Medicine Department of Radiology AnkaraTurkey $^{I}$

Objective: To assess the diagnostic efficiency of apparent diffusion coefficient $(\mathrm{ADC})$ values in differentiating schwannomas and meningiomas of the posterior fossa.

Materials and Methods: A total of 56 patients who referred for MRI of posterior fossa extra-axial lesions were retrospectively enrolled in our study. The mean ADC values of the tumor and reference value from contralateral cerebellar parenchyma obtained by using ROI from ADC maps by two radiologists in consensus. ADC ratios were calculated. Differences between schwannomas and meningiomas in mean ADC, ADC ratio values were statistically analyzed.

Results: $52.7 \%$ of patients were male, $47.3 \%$ were female with a mean age of 53.4. Based on conventional MRI features, 29 was interpreted as schwannoma, 26 as meningioma. Mean ADC values (x10-3 mm2 s-1) 
of schwannomas and meningiomas were $1.087 \pm 0.224$ and $0.729 \pm 0.130$, respectively. Mean ADC ratios were $1.56 \pm 0.34$ for schwannomas and $1.08 \pm 0.17$ for meningiomas. Mean ADC values and ADC ratios of schwannomas were significantly greater than meningiomas $(\mathrm{p}<0.001)$. When the diagnostic value of ADC was compared, the area under the curve between two tumor types was 0.957 , the sensitivity 0.92 , the specificity was $0.89(\mathrm{p}<0,001)$. Values for ADC ratios were found respectively $0.932,0.92$ and 0.79 between the two groups $(\mathrm{p}<0,001)$.

Conclusions: $\mathrm{ADC}$ value and $\mathrm{ADC}$ ratios may lead to the discriminating schwannomas and meningiomas. Although schwannoma and meningioma diagnose was based on typical imaging features, $\mathrm{ADC}$ value and ratio can provide clue for a discrepancy in controversial cases.

1056-THE ROLE OF HISTOGRAM ANALYSIS IN THE DIAGNOSIS OF CHOLESTEATOMA

$\underline{\text { Nursel Yurttutan }}{ }_{-}^{1}$, Nagihan Bilal ${ }^{2}$, Betül Kızıldağ ${ }^{1}$, Adem Doğaner ${ }^{3}$

Kahramanmaraşs Sütçüimam University Radiology KahramanmaraşTurkey ${ }^{I}$ Kahramanmaraş Sütçüimam University Otolaryngology Kahramanmaraş-Turkey ${ }^{2}$ Kahramanmaraş Sütçüimam University Biostatistics Kahramanmaraş Sütçüimam University-Turkey ${ }^{3}$

Purpose: The aim of this study is to investigate whether the histogram analysis, which will be performed on the temporal bone high resolution computed tomography (HRCT) examination obtained before the operation, contributes to the differentiation of cholesteatoma (CHS) and noncholesteatoma (NCHS).

Methods: In this study, 77 temporal bone HRCT examinations obtained preoperatively were evaluated. Histogram analysis was performed on the XML files obtained after blinded measurements made at the epitimpanyum level with standard size region of interest (ROI) using R Project 3.3.2 software. Mean, maximum, median, minimum, Standard Deviation (SD), variance, skewness, kurtosis, uniformity and entropy parameters were evaluated. Threshold values were found with ROC analysis and the level of diagnostic efficiency of these parameters in CHSNCHS distinction was determined.

Results: According to the surgical and histopathological evaluation, 41 CT scans in the CHS group and $36 \mathrm{CT}$ scans in the NCHS group were evaluated. A statistically significant difference was found between the CHS and NCHS groups in mean, maximum and median among the 10 histogram analysis parameters $(\mathrm{P}=0.036, \mathrm{P}=0.006, \mathrm{P}=0.043)$. When the ROC curve obtained for the mean of these parameters was examined, the area under the curve was 0.638 , and when the threshold value was chosen as 42.55 , the sensitivity of mean value in CHS-NCHS distinction was calculated as $86.50 \%$ and specificity as $56.10 \%$.

Conclusion: Thanks to computed tomography histogram analysis, more detailed data can be obtained with advanced analysis of pixels in cases where there is no use of additional radiation and contrast material by avoiding the repetition of imaging methods. In cases with MRI contraindications, more data can be obtained by histogram analysis on existing images to reduce the rate of false positive-negative situations in small lesions that are difficult to detect and characterize due to resolution problems.

1059-PREVALENCE OF ISOLATED THIRD WINDOW ABNORMALITIES IN CHILDREN WITHOUT SYMPTOMS

Fatma Ceren Sarioglu ${ }^{1}$ - Yeliz Pekcevik ${ }^{1}$, Handan Guleryuz ${ }^{2}$

Izmir Health Sciences University, Tepecik Education and Research Hospital Radiology Izmir-Turkey ${ }^{1}$ Dokuz Eylul University School of Medicine Radiology Izmir- ${ }^{2}$

Purpose: Our aim was to determine the rate of isolated third window abnormalities (TWA) in asymptomatic children on computed tomography (CT) images.
Materials and Methods:Pediatric emergency department data was reviewed retrospectively to identify children who had temporal bone CT scans between 2016 to 2020 . Patients with vestibular and/or auditory symptoms $(n=27)$ were excluded and 68 children who were presented with other causes such as trauma or facial paralysis were accepted as the asymptomatic group. TWA were categorized as superior semicircular canal dehiscence (SSCD), posterior semicircular canal dehiscence (PSCD), enlarged vestibular aqueduct (EVA), perilymph fistula (PLF). Two pediatric radiologists evaluated TWA bilaterally and classified as present or absent. Symptomatic group was determined by scanning the archive of otorhinolaryngology department to identify children who had temporal bone CT scans with vestibular and/or auditory symptoms. Patients with congenital cochleovestibular anomalies $(n=39)$ were excluded. Both groups were similarly interpreted, and the rate of the isolated TWA was calculated for each group.

Results:The prevalence of isolated TWA was found as $4.4 \%$ (6 of 136 ears) and $10.2 \%$ (37 of 362 ears) in 68 asymptomatic children and 181 symptomatic children, respectively $(\mathrm{p}=0.048$ ). There were no children with EVA or PLF in the asypmtomatic group. The rate of the EVA was significantly different between the groups $(\mathrm{p}=0.005)$ while the remainders showed no differences.

Conclusions: The rate of the isolated TWA was found different between the symptomatic and asymptomatic children. We suggest that asymptomatic children should be evaluated for vestibular and audological issues.

\section{0-COMPARISON OF CONVENTIONAL AND ELASTOGRAPHIC ULTRASOUND FINDINGS IN SUBACUTE THYROIDITIS}

$\underline{\text { Veysel Kaplanoğlu }}{ }^{1}$, Özlem Güngör ${ }^{1}$

Keçiören training and research hospital Radiology ANKARA-Turkey ${ }^{1}$

Purpose:We aimed to compare B-mode, color Doppler ultrasound (CDU), superb microvascular imaging (SMI) and elastography [strain elastography(SE) and shear wave elastography(SWE)] findings in the diagnosis of subacute thyroiditis (SAT).

Method:In this retrospective study, we evaluated 30 patients with SAT (SAT group) and 30 healthy participants (control group). All clinical and laboratory data were collected from electronic health record system. Quantitative values of B- mode (echo), CDU-SMI (resistive index, RI; pulsatility index, PI; power vascular index, PVI; SMI vascular index, SVI), SE (strain index - round ROI, TRSI; manual ROI, TMSI) and SWE [round(R) ROI, manual(M) ROI, parenchyma/muscle ratio(r) and kilopascal $(\mathrm{k})$, meters per second(m)] of the thyroid right and left lobe parenchyma in the SAT and control groups were obtained from archive of digital images from US device. We compared these values in the SAT group and between the SAT and control groups.

Results:The capacity of US values in predicting presence of SAT were analyzed using ROC curve analysis. In the right thyroid lobe (cutoff;AUC; sensitivity;specificity) $\operatorname{TRk}(\geq 33.75 ; 0.997 ; 100 ; 96.7)$, TRrk $(\geq 0.8515 ; 0.823 ; 70 ; 73.3), \operatorname{TMk}(\geq 48.6 ; 1.100 ; 100)$, TMrk $(\geq 0.7565 ; 0.851 ; 80 ; 73.3), \operatorname{TRm}(\geq 3.09 ; 0.998 ; 100 ; 96.7)$, $\operatorname{TRrm}(\geq 0.861 ; 0.855 ; 75 ; 70)$, TMm( $(\geq 3.75 ; 1 ; 100 ; 100)$, $\operatorname{TMrm}(\geq 0.8835 ; 0.82 ; 70 ; 80), \operatorname{RI}(\geq 0.635 ; 0.85 ; 80 ; 93.30)$, $\mathrm{PI}(\geq 0.89 ; 0.893 ; 8580), \mathrm{SVI}(\leq 5.745 ; 0.718 ; 70 ; 70 ; \mathrm{p}=0.009)$ values and in the left thyroid lobe TRk $(\geq 38.2 ; 0.995 ; 100 ; 96.70)$, $\operatorname{TRrk}(\geq 0.7475 ; 0.852 ; 80 ; 73.30), \operatorname{TMk}(\geq 38.5 ; 0.997 ; 100 ; 93.30)$, $\operatorname{TMrk}(\geq 0.721 ; 0.865 ; 80 ; 73.30)$, TRm(3.145;0.97;95;90), $\operatorname{TRrm}(\geq 0.784 ; 0.828 ; 85 ; 73.30), \operatorname{TMm}(\geq 3.27 ; 0.987 ; 95 ; 93.30)$, $\operatorname{TMrm}(\geq 0.8525 ; 0.892 ; 80 ; 80), \operatorname{TMSI}(\geq 1.08 ; 0.753 ; 65 ; 73.30 ; \mathrm{p}<0.03)$, $\mathrm{RI}(\geq 0.585 ; 0.941 ; 90 ; 90), \mathrm{PI}(\geq 0.865 ; 0.919 ; 95 ; 86.70)$ values were found to have a diagnostic value in predicting SAT $(\mathrm{p}<0.001)$.

Conclusion: Quantitative parameters of SWE may provide better diagnostic distinction in tissue stiffness than SE in SAT. Quantitative parameters of SMI and SWE can contribute to differentiating SAT from normal thyroid parenchyma. 


\section{1-IMAGING FINDINGS IN DEEP NECK INFECTIONS}

Merve Büyüker ${ }^{1}$, Fatma Can ${ }^{1}$, Gülgün Yllmaz Ovall ${ }^{1}$, Yüksel Pabușçu ${ }^{1}$ Celal Bayar Üniversitesi Tıp Fakültesi Radyoloji Anabilim Dalı ManisaTurkey $^{1}$

Purpose: Deep neck infection (DNI) is an infection of potential spaces and facial planes of the neck region. Despite advances in diagnostic testing and the availability of modern antibiotic therapy, these infections continue to be important causes of morbidity and mortality, especially when not treated early. Imaging methods play an important role in the diagnosis of DNI and its complications. Quick diagnosis and selection of the correct imaging method are important. Computed Tomography (CT) is the first method of choice to show the spread of infection and the complications it causes. In our study, we aimed to present imaging findings of etiologic features, spreading and complications of cases presenting with DNI.

Method: 68 adult age group patients diagnosed with DNI by CT and / or Magnetic Resonance Imaging (MRI) in our clinic in the last 6 years were analyzed retrospectively. The following clinical features were analyzed and compared: age, sex, etiology, affected neck region and complications.

Presentation: 68 patients were included in our study. Compartments where DNI is spread; pharyngeal mucosal, parapharyngeal, retropharyngeal, visceral, parotid, submandibular, sublingual, massicator, buccal, carotid, perivertebral, anterior cervical and posterior cervical compartment. The most frequently affected areas; pharyngeal mucosal and submandibular compartments. DNI most frequently developed as a complication of tonsillitis and periapical abscess. Diabetes Mellitus was the most common comorbid disease in etiology. DNI developed after head and neck operation in 2 patients and tooth extraction in 7 patients. Mediastinal extension and sepsis were the most common lifethreatening complications. The mortality rate in our DNI patients was $5.9 \%$.

Result: It is important to define the anatomic distribution of the infection and the complications it causes in cases presenting with DNI. It is a guide in determining the drainage or medical treatment options in the presence of accompanying abscess. CT is the first and most preferred imaging method.

\section{2-INTERNAL CAROTID ARTERY STENOSIS ACCORDING} TO PLAQUE MORPHOLOGY

Gülen Burakgazi ${ }^{1},{ }^{\text {, Selen Aslan }}{ }^{2}$, Hande Melike Bülbül ${ }^{1}$, Fatma Beyazal Çeliker ${ }^{1}$

RTEÜ Radyoloji rize-Turkey ${ }^{1}$ MKÜ Radyoloji Hatay-Turkey ${ }^{2}$

Purpose:The risk of stroke can be determined by radiological methods by evaluating both the degree of luminal stenosis and the carotid plaque structure. Although stroke is the third most common cause of death in the population, it is the most common cause of disability in adults. Extracranial atherosclerotic disease accounts for $15-20 \%$ of all ischemic strokes and is a treatable cause of ischemic stroke. The degree of lumen stenosis is an important indicator of stroke risk. With the NASCET study, the benefit of endarterectomy in symptomatic patients with stenosis above $70 \%$ has been shown. However, the relationship between the content, growth, and increasing stenosis of the plaque with stroke and transient ischemic attack is complex. Therefore, the plaque structure and content may play an important role in the decision of surgical treatment in symptomatic patients with moderate stenosis and in patients with asymptomatic stenosis $(1,2)$. Carotid Doppler USG continues to be the first noninvasive imaging modality for carotid artery stenosis. However, CTA is an excellent auxiliary technique in the preoperative evaluation of carotid diseases, and it significantly affects the decisions regarding operation treatment $(3,4)$. CTA is an imaging method that enables 3 -dimensional images to be obtained on the basis of X-rays. Our aim in this study is to compare the degree of stenosis detected in Doppler USG and CTA according to the plaque type. Thus, it is aimed to determine in which plaque types and degrees of stenosis can be recommended further examination with CTA.

Material and methods: The examinations of 101 (67 M, $34 \mathrm{~K})$ cases who had both Doppler USG and CTA tests between January 2018 and January 2019 were evaluated retrospectively. In both examinations, measurements were made from the proximal internal carotid artery (ICA) and divided into five groups according to the degree of stenosis. Again, in both examinations, the plaques were evaluated in three groups as soft, calcific and mixed types.

Results: Kappa analysis was performed in order to determine the agreement of Doppler and ICA in determining the stenosis percentage and plaque type, since the gold standard diagnostic test (DSA) was not used in the study. Right ICA and right vertebral artery Doppler USG and CTA showed stenosis degrees independent of plaque type ( $\mathrm{p} 0.00$ ).

In addition, it was observed in Doppler examination that calcific plaque could cause diagnostic difficulty in determining the level of stenosis, and the correlation of calcific and mixed plaques with other cases was also evaluated with Kappa analysis. Right ICA doppler USG and CTA were compatible in determining the types of mixed plaques $(p=0.00)$. Right ICA doppler USG and CTA were found to be compatible in determining soft plaque types ( $\mathrm{p}$ $=0.003)$. Left ICA doppler USG and CTA were found to be compatible in determining soft plaque types $(p=0.02)$. Doppler USG and CTA examinations were more compatible in calcific plaque types.

Discussion and Conclusion: Although intra-arterial digital subtraction angiography (DSA) is the gold standard method for determining the degree of arterial stenosis, non-invasive examination methods such as Doppler USG, CTA and MRI are preferred in many centers to evaluate plaque morphology as well as stenosis. Determining the degree of stenosis and plaque types with Doppler USG and Computed Tomography helps to plan the treatment.

1106 -DIAGNOSTIC VALUE OF SWE IN THE EVALUATION OF AUTOIMMUNE THYROIDITIS

Mustafa Devran Aybar ${ }^{1}$, Önder Turna ${ }^{2}$

Istanbul Private Medigold Sultan Hospital radiology istanbul-Turkey ${ }^{1}$ Mehmet Akif Cardiovascular and Thoracic Surgery Training and Research Hospital radiology istanbul-Turkey ${ }^{2}$

Purpose: The aim of this study is to assess the applicability of shear wave elastography (SWE) in the diagnosis of autoimmun thyroiditis (AT) patients.

Methods and Materials: The study group consisted of 64 patients with first-diagnosed AT and 68 control subjects (CS). In all patients with AT and CS, sonoelastographic measurements were made in both thyroid lobes. The mean SWE values were recorded using $\mathrm{m} / \mathrm{s}$ and $\mathrm{kPa}$ as units. ROC curve analysis was performed and diagnostic accuracy of SWE values were evaluated.

Results: The mean SWE values of AT $(2.57 \pm 0.58 \mathrm{~m} / \mathrm{s}$ and $19 \pm 5.2 \mathrm{kPa})$ were significantly higher than the values of CS $(1.59 \pm 0.41 \mathrm{~m} / \mathrm{s}$ and 11 $\pm 3.9 \mathrm{kPa})(\mathrm{p}=0.006$ and $\mathrm{p}=0.001$, respectively). ROC curve analysis showed high diagnostic accuracy for differentiating of AT from CS with SWE values (AUC 0.964 [\%95 CI, 0.931-0.973] in $\mathrm{m} / \mathrm{s}$ and AUC 0.977 [\%95 CI, 0.951-0.985] in $\mathrm{kPa}$ ). Using SWE cut-off values as $1.98 \mathrm{~m} / \mathrm{s}$ and $15.3 \mathrm{kPa}$, sensitivity and specificity were $86.1 \%, 84.9 \%$ and $88.8 \%, 89.7 \%$, respectively.

Conclusion: SWE is a useful imaging method that can be used with routine ultrasonography in evaluation of the AT. 
1111-MULTİPARAMETRIC US IN PARATHYROID PATHOLOGIES: B MODE, CDU AND ELASTOGRAPHY

Veysel Kaplanoğlu ${ }^{1}$, Arzu Or Koca ${ }^{2}$, Cansu Öztürk ${ }^{1}$, Özlem Güngör ${ }^{1}$, Tolga Akkan ${ }^{2}$, Gülçin Șimșek ${ }^{3}$, Aydın Acar ${ }^{4}$, Mustafa Altay ${ }^{2}$, Selma Uysal Ramadan ${ }^{1}$

Keçiören training and research hospital Radiology ANKARATurkey ${ }^{I}$ Keçiören training and research hospital Endocrinology and Metabolism ANKARA-Turkey ${ }^{2}$ Keçiören training and research hospital Pathology ANKARA-Turkey ${ }^{3}$ Keçiören training and research hospital Otorhinolaryngology ANKARA-Turkey ${ }^{4}$

Purpose:We aimed to evaluate the different measurement techniques performed by Color Doppler ultrasonography (CDU), Superb Microvasculer Imaging (SMI), Strain Elastography (SE) and Share View Elastography (SWE) in parathyroid lesion (PL) localization, in comparison with thyroid parenchyma.

Methods:Parathyroid lesions were detected on imaging in 97 cases (prospective). Of these 97 cases, 103 lesions (100 adenomas, 3 hyperplasias) confirmed by the operation were included in the study. Forty patients with normal thyroid US and hormone values were included as the control group. B-mode (echo), CDU and SMI, SE, SWE were performed in all cases and quantitative values, stated below, were recorded; CDU and SMI: resistive-pulsatility-vascular index (RI, PI, power VI (PVI), SMIVI (SVI), SE: longutidinal (L)-transvers (T) axle strain index (round (R) ROI (LRSI,TRSI))-manual (M) ROI (LMSI,TMSI)), SWE: L-T axle, kilopaskal (k)-meter/second (m), R-M-ROI and lesion/muscle ratio (r). The quantitative values, obtained, were compared with each other, thyroid parenchyma, and pathological results.

Results: In the parathyroid lesion, on the L; T axis were compared with each other $(\mathrm{p}=0)$ and PL with TP (PL;TP;p), a significant difference was found in the median values of SWE. In respectively; PL-kR $(14,9 ; 11,4)$, PL-kRr $(0,45 ; 0,63)$, PL-mR $(2,12 ; 1,93)$, PL-mRr $(0,65 ; 0,77)$, PL-kM $(15,65 ; 11,5)$, PL-kMr $(0,45 ; 0,63)$, PL-mM $(2,17 ; 1,86)$, PL-mMr $(0,65 ; 0,78)$ and respectively $\operatorname{LkR}(14,9 ; 19,95 ; \mathrm{p}=0,007), \mathrm{LkRr}$ $(0,45 ; 0,52 ; \mathrm{p}=0,048), \operatorname{LmR}(2,12 ; 2,43 ; \mathrm{p}=0,009), \mathrm{LmRr}$ $(0,66 ; 0,73 ; \mathrm{p}=0,078), \mathrm{LkM}(20,35 ; 15,65 ; \mathrm{p}=0,008), \mathrm{LkMr}$ $(0,45 ; 0,53 ; p=0,045), \operatorname{LmM}(2,17 ; 2,54 ; p=0,002), \mathrm{LmMr}$ $(0,66 ; 0,74 ; \mathrm{p}=0,024)$. Significant difference was found in PVI $(25.7 ; 14.75)$ and SVI $(15.82 ; 8.18)$ values $(\mathrm{p}<0.001)$.

In ROC analysis of PL according to TP (CutOff;AUC;p) in SWE; LkR $(<17,75 ; 0,648 ; 0,007), \mathrm{LkRr}(<0,48 ; 0,608 ; 0,048), \mathrm{LmR}$ $(<2,36 ; 0,642 ; 0,009)$, LkM $(<18,45 ; 0,645 ; 0,008), \mathrm{LkMr}$ $(<0,50 ; 0,609 ; 0,045), \mathrm{LmM}(<2,40 ; 0,669 ; 0,002), \mathrm{LmMr}$ $(<0,71 ; 0,625 ; 0,022)$, PVI $(>18,45 ; 0,743 ;<0.001)$ in CDU and SVI $(>9.95 ; 0.807 ;<0.001)$ values in SMI were determined.

Conclusion:Share view elastography measured with different techniques in detecting parathyroid lesions, quantitative parameters provide better lesion differentiation in tissue stiffness and vascular index values in tissue vascularization.

\section{1-FIRST IMPRESSIONS AND IMMEDIATE RESULTS WITH NEW LVIS EVO STENT}

$\underline{\text { Yilmaz Onal }}^{1}$, Murat Velioglu ${ }^{1}$

FSM Training and Research Hospital Radiology Istanbul -Turkey

Purpose:To assess both technical feasibility and immediate results with Lvis Evo assisted embolization of intracranial aneurysms.

Material-Method: We identified the patients with intracranial aneurysms treated with Lvis Evo stent-assisted coiling. Immediate postoperative angiographic outcomes and periprocedural complications of the procedures were recorded.

Results: 5 stent-assisted embolization procedures were performed using the same stent. Localization of the aneuryms were at basilar tip (2), MCA bifurcation (1), Acom (1) and PCA (1). Technical success was \%80. In a patient with a wide-necked complex MCA bifurcation aneurysm, stent that was attempted to be deployed from the lower incorporated branch by the aneurysm could not be successfully fully opened in that sharp angle. Immediate occlusion rate was $\% 60$.

Conclusion: Low profile Lvis Evo stents could become a useful tool in the treatment of intracranial anuerysms. Although the number of our patients is small, we think that in wide necked bifurcation aneurysms the new Lvis Evo stent can reduce the need for $\mathrm{Y}$ stent assistance for protecting daughter vessels by bulging into the aneurysm neck with high radial force. Studies with larger groups of patients are needed to assess the true outcome of this stent.

\section{6-INTRAARTERIAL T PA INJECTION WHILE STENT RETRIEVER DEPLOYED IN ACUTE STROKE}

YUNUS YILMAZSOY ${ }^{1}$, MUSTAFA HIZAL $^{2}$ BOLU ABANT IZZZET BAYSAL UNIVERSITY Radiology BOLU-Turkey ${ }^{1}$ BOLU ABANT IZZET BAYSAL UNIVERSITY Radology BOLU-Turkey ${ }^{2}$

Purpose: Tissue plasminogen activator (t-PA) has been used in many studies in the treatment of stroke by both intraarterial and intravenous access. In this study, we shared our experience on the use and results of $\mathrm{t}$-PA administered through the distal access catheter as a salvage therapy in the presence of partial flow obtained while the stent retriever was deployed.

Material and Method: We retrospectively analyzed the radiological and clinical information of the patients who applied to our hospital between January 2019 and September 2020 due to acute stroke, despite the repeated mechanical thromboaspiration procedure, whose vascular patency could not be achieved, and therefore, who were applied intra-arterial tPA while the stent retriever was deployed and allowed flow in the occluded segment as a salvage therapy.

Results: After a mean of 3.35 failed mechanic thrombectomy procedures intraarterial t-PA administered via distal access catheter while stent retriever deployed. With this technique, Thrombolysis in cerebral infarction (Tici) $2 b, 2 c$ or 3 scores were obtained in $71.4 \%$ of the 14 patients.

Conclusion: As a result, satisfactory recanalization rates and no major complications suggest that the technique is safe. However, while selective intraarterial t-PA should be administered, the onset of stroke and the duration of the procedure, blood pressure, extent of the infarct area, and the previous use of anti-thrombolytic agents should be considered

\section{7-Y STENT ASSISTED COILING OF RUPTURED WIDE NECK INTRACRANIAL ANEURYSM}

İsmail Okan Yildırım ${ }^{1}$, Mehmet Kolu ${ }^{2}$, Mehmet Akif Durak ${ }^{3}$, Bora Tetik $^{3}$, Ramazan Paşahan ${ }^{3}$, Serkan Sevgi ${ }^{1}$, Şükrü Gürbüz ${ }^{4}$, Kaya Saraç $^{1}$

Inonu University School of Medicine, Department of Radiology MalatyaTurkey ${ }^{1}$ Harran University School of Medicine, Department of Radiology Şanlufa-Turkey ${ }^{2}$ Inonu University School of Medicine, Department of Neurosurgery Malatya-Turkey ${ }^{3}$ Inonu University School of Medicine, Department of Emergency Malatya-Turkey ${ }^{4}$

Purpose:The objective of the present study is to analyze the outcomes of patients with subarachnoid hemorrhage (SAH) in the acute phase after treatment with Y-stent-assisted coiling (YSAC) embolization.

Methods: This retrospective study assessed of 30 patients with acutely ruptured wide-neck aneurysms following YSAC treatment between April 2013 and October 2019. The demographic data, aneurysm occlusion grade, procedural and periprocedural complications, and clinical outcomes were assessed.

Results: The procedure was completed in 30 cases $(90.1 \%)$ and technical failure occurred in 3 cases $(9.1 \%)$. Immediate control angiography revealed that total occlusion Raymond-Ray Class 1 (RR1) was achieved 
in $21(70 \%)$, neck filling (RR2) in eight (26.6\%) and sac filling (RR1) in one $(3.3 \%)$ aneurysm. Upon angiographic follow-up, RR1 occlusion was observed in $15(71.4 \%)$ patients, RR2 in three (14.3\%) patients and RR3 in three $(14.3 \%)$ patients. In-stent thrombus developed in five $(16.6 \%)$ patients; procedural ischemic events were observed in four $(13.3 \%)$ patients; and two $(6.6 \%)$ patients were symptomatic. A periprocedural asymptomatic intracranial hemorrhage was detected in two patients. At discharge, $17(56.6 \%)$ patients were in good clinical condition, six $(20 \%)$ were in a severe disability condition, and seven (23.3\%) patients had died. At the final follow-up visit (mean: 18.9 months), 16 (76,2\%) of 21 patients were in a good clinical condition and five $(23.8 \%)$ had severe disabilities.

Conclusions: Treating ruptured complex wide-neck aneurysms in SAH patients during the acute phase with the YSAC procedure can be challenging. The YSAC procedure is a viable, safe and effective approach for this patient group.

\section{4-PREDICTORS OF FUTILE RECANALIZATION FOLLOWING MECHANICAL THROMBECTOMY}

Orkun Sarioglu ${ }^{1}$, Fatma Ceren Sarioglu ${ }^{2}$, Umit Belet ${ }^{2}$

Izmir Democracy University Radiology Izmir-Turkey ${ }^{1}$ Health Sciences University, Izmir Tepecik Education and Research Hospital Radiology Izmir-Turkey $^{2}$

Purpose:The effectiveness of mechanical thrombectomy (MT) in acute ischemic stroke (AIS) is demonstrated by important trials. However, good clinical results are not always observed even after a successful recanalization, which is described as 'futile recanalization'. Our aim was to evaluate the variables associated with futile recanalization.

Materials and Methods:Patients who underwent MT in our hospital between January 2017 and July 2020 were retrospectively reviewed and subjects with unsuccessful recanalization were excluded. Patients were divided into two groups according to the modified Rankin Scale (mRS) scores at 3 months as good outcome $(\mathrm{mRS} \leq 2)$ and bad outcome $(\mathrm{mRS}>2)$. Demographic, clinical, and periprocedural features were compared between the groups, and ROC curves were made. Logistic regression analysis was performed to analyze the independent predictors of the futile recanalization.

Results:Sixty-eight patients (33 female, 35 male; mean age $63.29 \pm$ 14.90) were included in the study. Futile recanalization was observed in $22(32.4 \%)$. Patients with futile recanalization had higher initial NIHSS $(\mathrm{p}=0.003)$ and lower ASPECT scores $(\mathrm{p}=0.014)$, had longer intervals from onset to puncture $(\mathrm{p}=0.026)$, experienced first pass recanalization less frequently $(\mathrm{p}=0.024)$, and had a higher rate of symptomatic hemorrhage $(\mathrm{p}<0.001)$. In the logistic regression analysis, the time from onset to puncture of $>235$ minutes and symptomatic hemorrhage were found as independent predictors of futile recanalization.

Conclusion:Futile recanalization is a relatively common condition, particularly in patients with prolonged time to groin puncture and those who experienced symptomatic hemorrhage after the treatment.

\section{3-DIFFUSION TENSOR MAGNETIC RESONANCE IMAGING IN MULTIPLE SCLEROSIS PATIENTS}

$\underline{\text { Kaan Alişar }}{ }^{1}$, Ebru Özan Sanhal ${ }^{2}$, Gökçe Kaan Ataç ${ }^{3}$, Refah Sayın ${ }^{4}$ Erzurum Regional Training and Research Hospital Radiology ErzurumTurkey ${ }^{1}$ Antalya Training and Research Hospital Radiology AntalyaTurkey ${ }^{2}$ Ufuk University School of Medicine Radiology Ankara-Turkey ${ }^{3}$ Ufuk University School of Medicine Neurology Ankara-Turkey ${ }^{4}$

Purpose: To quantitatively determine microstructural changes in normal appearing white matter areas of MS patients using Diffusion Tensor MRI.

Materials and Methods: In this study, 15 patients and 15 control subjects were evaluated by Diffusion Tensor MRI. Fractional Anisotropy (FA) and Diffusion Coefficient (ADC) measurements were made by using region-of-interest (ROI) method of 32 plaques, normal appearing white matter areas (NAWM) from the peri-plaque and symmetrical white matter of the patient group with corpus callosum, periventricular and centrum semiovale white matter areas of both patient and control groups and statistical analysis was performed.

Results: There was a significant FA decrease in corpus callosum and centrum semiovale in patient group. In the NAWM areas around the plaque, there is a low FA value compared to the symmetry of the plaque in the contralateral hemisphere. Significant height increase of the corpus callosum was present in the patient group during ADC measurements. In genu both FA and ADC, in splenium FA and in periventricular NAWM $\mathrm{ADC}$ asymmetries was observed in the patient group. As the EDSS score increased, a significant decrease in FA value was detected in the genus. In addition, cut-off value was determined in corpus callosum in order to predict healty individuals.

Conclusion: Quantitative analysis of DTI data revealed abnormalities in the NAWM of MS patients. DTI can be used as a predictive indicator of clinical outcome and response to treatment in the early detection of white matter damage and prospective studies of MS patients. For patients with suspicion of MS, the diagnosis can be supported or excluded by the addition of short-term sequence for DTG to routine MRI protocols and determining white matter cut-off DTG quantitative values to be obtained by carrying out studies with higher populations.

\section{8-EVALUATION OF ANTERIOR THALAMIC RADIATION WITH DTI IN NF1}

\section{DILEK HACER CESME ${ }_{\text {- }}^{\text {- }}$}

|Bezmialem Vakif University Radiology Istanbul-Turkey ${ }^{\text {}}$

Purpose: Executive functional disorders among neurocognitive functions are common in patients with NF1. The ADC and FA values of anterior thalamic radiation, which plays a role in the inhibitory control mechanism, were investigated whether there was a difference between the NF1 patients with and without UBO and the healthy control group.

Method: Thirty children followed up with a diagnosis of NF1 and 17 age-matched children were included in the study. The mean age of 30 children with NF1 was $10.47 \pm 5.43$, while it was $11.35 \pm 5.43$ in the healthy control group. They were divided into two groups as those with and without hamartomas (UBOs) with conventional MRI findings. Comparison was made by measuring $\mathrm{ADC}$ and $\mathrm{FA}$ values from Anterior thalamic radiation in patients with NF1 and healthy controls.

Results: There was a significant difference in terms of ADC and FA values obtained from anterior thalamic radiation (respectively; $\mathrm{p}$ : 0.0001, p: 0.0001) in patients with NF1 compared to healthy controls. FA values decreased significantly as ADC values increased. There was a significant difference between the patients with NF1 with and without UBOs and the healthy control group in terms of anterior thalamic radiation ADC and FA values (p: 0.0001 for each). There was no significant difference in anterior thalamic radiation ADC and FA values between cases with and without UBOs.

Conclusions: There are ADC and FA changes in ATR in patients with NF1. It was concluded that microstructural level white matter damage in ATR in children with NF1 is strongly associated with inhibitory control mechanism disorders from executive functions. We think that the prediction of executive functions and other neurocognitive disorders due to the impairment of white matter integrity in cases with NF1 can be made with DTI.

1009-TEMPORAL LOBE PERIVASCULAR SPACES RADIOLOGICAL MORPHOLOGY AND FOLLOW UP FINDINGS $\underline{\text { Rifat Ozpar }}{ }^{1}$, Gokhan Ongen ${ }^{1}$, Mufit Parlak ${ }^{1}$, Bahattin Hakyemez ${ }^{1}$ Bursa Uludag University Faculty of Medicine Radiology Bursa-Turkey ${ }^{l}$

Purpose: Perivascular spaces located in the temporal lobe are distinct from other perivascular spaces in the central nervous system because of 
the frequently encountered perilesional hyperintensity $(\mathrm{PH})$ in magnetic resonance imaging (MRI), and thus may be misdiagnosed as a tumoral mass. The aim of this study was to evaluate morphologic and follow-up findings of the temporal lobe perivascular spaces (TLPS) and lookup for a possible relationship between them.

Methods: 21 patients with TLPS diagnosed and followed-up with MRI and with no additional treatment during follow-up were included in this study. Count, long-axis, and short-axis diameters of the lesions, presence of $\mathrm{PH}$, and short-axis diameter of the $\mathrm{PH}$ were measured in both initial and follow-up MRI examinations. The follow-up duration for each patient was determined. Changes in the measured parameters and potential relationships between them were investigated.

Results: The mean duration of the radiological follow-up was 20.9 \pm 22.6 (3-89) months. PH was present around $76.2 \%$ (16/21) of the lesions. Lesions and PH were stable in $71.4 \%(15 / 21)$ of the patients. Lesion progression without $\mathrm{PH}$ was observed in $9.6 \%(2 / 21)$ of the patients, partial regression of the lesion in $4.8 \%(1 / 21)$, partial regression of the $\mathrm{PH}$ in $9.6 \%(2 / 21)$, and complete regression of lesion and $\mathrm{PH}$ in $4.8 \%$ (1/21). No significant relationships were found between count, long-axis, and short-axis of the lesion, short-axis of the $\mathrm{PH}$, and course of the TLPS.

Conclusion: TLPS is usually stable, but sometimes changes within lesion and $\mathrm{PH}$ may be observed during follow-ups. Regression of the $\mathrm{PH}$ in some patients indicates that $\mathrm{PH}$ may reflect interstitial edema more than gliosis. $\mathrm{PH}$ is an independent feature than lesion size. Further investigations are required to understand the pathophysiology of the TLPS with PH.

\section{5-INCIDENTAL WHITE MATTER HYPERINTENSITIES IN} HEALTHY 18 TO 55 YEAR OLD INDIVIDULAS

\author{
$\underline{\text { Nimet Akın }}{ }^{1}$, Banu Topçu Çakır ${ }^{1}$ \\ Ankara City Hospital Radiology Ankara-Turkey ${ }^{I}$
}

Purpose:White matter hyperintensities; although it is common especially in elderly individuals, it is one of the MRI findings that is frequently detected in asymptomatic individuals with no known disease at any age. Incidental white matter hyperintensity without a detected cause can be extremely distressing for especially young patients. Thus, it is of utmost importance to determine the natural course of white matter hyperintensity with an undetected cause and its relation to neurologic symptoms. To solve this problem, the prevalence and distribution of white matter hyperintensity with undetected causes should be investigated first. The aim of this retrospective study was to investigate prevalence of white matter hyperintensities that could not be attributed to any cause in the age range we determined and the characteristics of the white matter hyperintensities we detected.

Materials and Methods: The study was retrospective and was carried out by evaluating 1000 Brain MRI examinations that were taken between 01.02.2020-29.02.2020 at Ankara City Hospital Radiology Clinic and met the inclusion criteria. The device used for Brain MRI was determined in the individuals included in the study. The clinical symptoms of the individuals included in the study or the reason for performing the examination were learned from the information in the system and recorded. The gender and ages of the individuals were noted. Two different age groups were formed as 18-35 and 36-55. The parameters we look at in brain MRI images; it was examined on the axial FLAIR sequence, which is one of the conventional sequences, and it was first noted whether there was a hyperintense focus in the white matter. Subsequently, in individuals with white matter hyperintensity; the number, size and localization of white matter hyperintensities were investigated. Finally, it was evaluated whether the patients had previous examinations in our system and if there were any changes in the detected white matter hyperintensities.

Results: In our study, we found the prevalence of white matter hyperintensity $40.3 \%$ in individuals aged $18-55$ who meet the inclusion criteria. The prevalence of white matter hyperintensity increased with age in both women and men. When female and male individuals between the ages of 18-35 and 36-55 are compared; the prevalence of white matter hyperintensity was higher in women in both groups. However, no statistical relationship was found between gender and the presence of white matter hyperintensity in both age groups. Among the individuals with white matter hyperintensity, $25.6 \%$ had 1 foci, $17.9 \%$ had 2 foci, $7.7 \%$ had 3 and $48.9 \%$ had more than 3 foci. The number of lesions increased with increasing age. However, there was no statistical relationship between gender and the number of white matter hyperintensities detected. In individuals with white matter hyperintensity (except those with multiple hyperintense foci) the size is less than $2 \mathrm{~mm}$ in $33.5 \%$, between $2 \mathrm{~mm}$ and $5 \mathrm{~mm}$ in $9.2 \%$ and greater than $5 \mathrm{~mm}$ in $0.2 \%$ hyperintense focus was encountered. Within the scope of the research, a total of 838 different white matter hyperintensities were determined and the localization with the most hyperintense focus; it was determined as the frontal deep white matter level. There was no statistical relationship between the complaints of individuals coming to the clinic and the presence of white matter hyperintensity.

Conclusion: The data we obtained in our study; we think that it can help us and will contribute to future studies, in the differential diagnosis of white matter hyperintensities that may accompany any pathology and white matter hyperintensities without clinical significance and in understanding the pathogenesis and clinical significance of incidental white matter hyperintensities in healthy individuals.

\section{6-CEREBRAL MR PERFUSION FINDINGS IN DIABETIC PATIENT GROUPS}

İbrahim İlik ${ }^{1}$, Harun Arslan ${ }^{2}$, Adem Yokuș ${ }^{2}$, Muhammed Batur ${ }^{3}$, Rıfkı Üçler ${ }^{4}$, Hüseyin Akdeniz ${ }^{2}$

Van Training and Research Hospital Department of Radiology VanTurkey ${ }^{I}$ Van Yüzüncü Yll University Department of Radiology VanTurkey ${ }^{2}$ Van Yüzüncü Yll University Department of Ophthalmology Van-Turkey ${ }^{3}$ Van Yüzüncü Yll University Department of Endocrinology and Metabolism Van-Turkey ${ }^{4}$

Purpose: We aimed to examine the relationship between diabetic patient groups and cerebral MR perfusion.

Method: 52 patients with type 2 diabetes mellitus and 39 healthy individuals were included in the study. The diabetic patients were divided into three groups as proliferative retinopathy(PRP), non-proliferative retinopathy(NPRP) and non-retinopathy(non-RP). The imaging processes were performed using a 1.5T MRI device. The images were obtained by DSC perfusion-weighted sequence. Cerebral blood flow(CBF) maps were created from raw data. Quantitative measurements of cortical grey matter were performed in the bilateral anterior-inferior frontal lobe, cingulate gyrus, anterior-medial temporal lobe, occipital lobe, and thalamus using region of interest(ROI). Besides, quantitative measurements were obtained from ipsilateral white matter as a reference. Afterwards, the grey matter $\mathrm{rCBF}$ values were proportioned to the white matter $\mathrm{rCBF}$ values.

Results: No statistically significant difference was found among the mean rCBF values of the three patient groups. When the study group and the healthy control group were compared, the $\mathrm{rCBF}$ values of the bilateral frontal lobe, cingulate gyrus, medial temporal lobe, thalamus and right occipital lobe were lower in the study group $(\mathrm{p}<0.05)$. There was no difference in the left occipital lobe and the left anterior temporal lobe ( $>0.05$ ). The rCBF values in the right anterior temporal lobe were lower in the study group $(\mathrm{p}=0.058)$.

Conclusion: There was no significant difference among the three diabetic patient groups. When compared with the healthy control group, regional hypoperfusion was generally observed in all lobes and a significant difference was observed in the majority of them. 
1018-PREDICTING PREOPERATIVE GRADE AND HISTOLOGICAL SUBTYPE OF MENINGIOMA MACHINE LEARNING

Ceylan Altintas Taslicay ${ }^{1}$, Elmire Dervisoglu ${ }^{1}$, Cengizhan Taslicay ${ }^{2}$, Ozgur Cakir ', Busra Bayrak Yaprak ${ }^{3}$, Cigdem Vural ${ }^{3}$

Kocaeli University School of Medicine Radiology Kocaeli-Turkey ${ }^{1}$ Istanbul Technical University Metallurgical and Materials Engineering Istanbul-Turkey ${ }^{2}$ Kocaeli University School of Medicine Pathology Kocaeli-Turkey ${ }^{3}$

Purpose: In this study, it was aimed to investigate the contribution of radiomics and machine learning (ML) to MR imaging in differentiating high-grade meningioma (HGM)-low grade meningioma (LGM) and classifying LGM as fibrous-nonfibrous.

Materials and Methods: The study protocol was approved by the institutional ethics committee.163 patients (32 HGM, 131 LGM; 15 fibrous, 116 nonfibrous) were included in this retrospective study. Preoperative MR images were evaluated using 14 semantic features. A total of 107 radiomics features were extracted from T2W, FLAIR, post-contrast T1W, post-contrast 3D T1-FFE, and ADC map images. Using semantic and radiomics features, five models were created to distinguish HGM from LGM. Classifications were made with support vector machine, logistic regression, k-nearest neighborhood, naive Bayes, decision tree, random forest, and neural network algorithms. The same ML algorithms were used to distinguish fibrous and nonfibrous meningiomas. Feature reduction was done with information gain method, and stratified 10-fold crossvalidation was used as an internal validation technique. The performance of ML algorithms was evaluated with the area under the curve (AUC), sensitivity, specificity, and accuracy rates. $p<0.05$ was considered statistically significant.

Results:The naive Bayes algorithm had the best diagnostic performance in all classification models. The ML model using the radiomics features obtained from post-contrast 3D T1-FFE images showed the best results in distinguishing HGM from LGM (AUC:0.906). All models using radiomics features showed better results than the model using semantic features alone. When radiomics features were added to semantic features, performances of ML algoritms were increased.In addition, classification using radiomics features obtained from all sequences had the highest performance in distinguishing LGM as fibrous and nonfibrous (AUC: 944).

Conclusions:ML using radiomics features in distinguishing HGM from LGM showed better results compared to semantic features. In addition, radiomics based ML showed promising results to distinguish LGM as fibrous and nonfibrous.

1019-PREDICTING IDH MUTATION IN GLIOBLASTOMA CONTRIBUTION OF RADIOMICS AND MACHINE LEARNING

Elmire Dervișoğlu ${ }^{1}$, Ceylan Altıntaș Tașlıçay ${ }^{1}$, Cengizhan Taşlıçay ${ }^{2}$, Burcu Alparslan $^{1}$, Ümay Kiraz ${ }^{3}$, Gür Akansel ${ }^{1}$

Kocaeli University Radiology Kocaeli-Turkey ${ }^{1}$ Istanbul Technical University Metallurgical and Materials Engineering Istanbul-Turkey ${ }^{2}$ Kocaeli University Pathology Kocaeli-Turkey ${ }^{3}$

Purpose: Isocitrate dehydrogenase (IDH) mutation is the most important independent molecular prognostic factor in glioblastoma (GBM). In this study, it was aimed to investigate the contribution of radiomics and machine learning to MR imaging in predicting IDH1 mutation in GBM.

Materials and Methods: 78 patients (15 IDH-mutant GBM, 63 IDHwild type GBM) were included in this retrospective study. Preoperative MR images were evaluated using Visually AcceSAble Rembrandt Images (VASARI) scoring. A total of 642 radiomics features, including shape-based, first, second and higher-order textural features, were extracted from T2W, FLAIR, post-contrast T1W, and ADC map images. Classifications were made with support vector machine, logistic regression, k-nearest neighborhood, naive Bayes, decision tree, random forest and neural network algorithms using VASARI features and radiomics features separately. Feature reduction was done with information gain method, and stratified 10-fold cross-validation was used as an internal validation technique. The performance of machine learning algorithms was evaluated with the area under the curve (AUC), sensitivity, specificity, and accuracy rates. McNemar's test was used to establish whether the classification with VASARI scoring is significantly different from classification with radiomics features. $\mathrm{p}<0.05$ was considered statistically significant.

Results: The neural network had the highest diagnostic performance in the classification made with both VASARI $(0.752$ AUC, $85 \%$ accuracy, $47 \%$ sensitivity, $94 \%$ specificity) and radiomics features (0.826 AUC, $87 \%$ accuracy, $60 \%$ sensitivity, $94 \%$ specificity). There was no statistically significant difference between the two classifications $(\mathrm{p}=0.803)$. The sensitivity of the naive Bayes algorithm using radiomics features $(87$ $\%)$ in detecting IDH1 mutation was significantly higher than the classification with VASARI features $(60 \%)(\mathrm{p}=0.002)$.

Conclusion: Machine learning using radiomics features showed higher sensitivity compared to VASARI scoring in determining IDH1 mutation in GBM.

\section{0-IODINE DENSITY MEASUREMENTS OF ACUTE INFARCTION ON DUAL ENERGY CT ANGIOGRAPHY}

Ekim Gumeler ${ }^{1}$, Ayça Akgöz Karaosmanoğlu ${ }^{1}$

Hacettepe University Radiology Ankara-Turkey ${ }^{1}$

Purpose:Dual energy computed tomography (DECT) is a relatively new state-of-art CT technology with emerging clinical applications in neuroimaging. In this study, we aimed to assess if there was any difference in iodine density measurements on DECT angiography of the acutely infarcted brain parenchyma due to middle cerebral artery (MCA) occlusion as compared to contralateral normally perfusing brain parenchyma.

Material and Method: Patients who had non-contrast head CT and DECT angiography of the head and neck with clinical suspicion of acute stroke were retrospectively reviewed. Among these, patients with MCA occlusion who also subsequently underwent magnetic resonance (MR) imaging after the aforementioned CT studies were identified and included in this study. DECT angiography images were processed on syngo.via V30 software and iodine maps were created for each patient. A neuroradiologist measured iodine density (ID) and iodine density percentage (ID\%) on iodine maps by drawing region of interests (ROI) on a single slice for each MCA vascular territory segment according to ASPECT scoring, with the exception of the internal capsule. Contralateral hemispheric measurements were also collected for comparison. Diffusionweighted brain MR images were used as a gold standard for assessment of the extent of acute infarction. Independent samples t-test and MannWhitney $U$ test were used for statistical analysis.

Results: There were eight patients $(\mathrm{M} / \mathrm{F}=4 / 4)$ (mean age=76.6) with MCA occlusion and had DECT angiographic imaging. Mean and median ID for regions of acute infarction and contralateral normal hemispheric brain parenchyma were $0.486 \pm 0.1719$ and $0.5 \mathrm{mg} / \mathrm{ml}, 1.407 \pm 4.4117$, and $0.6 \mathrm{mg} / \mathrm{ml}$, respectively. Mean ID\% for acute infarction and contralateral normal brain tissue were $8.63 \pm 3.25 \%$ and $10.89 \pm 2.7 \%$, respectively. Both ID (mean and median) and ID\% showed a significant difference among infarcted and normal brain tissue $(\mathrm{p}<0.005)$.Conclusion: ID and ID\% measurements on iodine maps on DECT angiography may be helpful in predicting regions of acute infarction in patients with acute MCA territory stroke.

\section{1-DIFFUSION WEIGHTED IMAGING MEASUREMENTS} OF CENTRAL SMELL REGIONS IN COVID 19 INFECTION

Veysel Burulday $_{-}{ }_{-}$, Nuray Bayar Muluk ${ }^{2}$, Mehmet Hüseyin Akgül ${ }^{3}$, Merve Sefa Sayar ${ }^{-}$ 
Van Training and ResearchVan Training and Research Hospital Hospital Radiology Van-Turkey ${ }^{I}$ Krrlkkale University, Faculty of Medicine ENT Kirikkale-Turkey ${ }^{2}$ Yuksek Ihtisas State Hospital Neurosurgery Kirikkale-Turkey ${ }^{3}$ Van Training and Research Hospital Infectious Diseasesand Clinical Microbiology Van- ${ }^{4}$

Purpose: This study was conducted to investigate the findings of neuroinvasion in central smell centers with Cranial MRI diffusionweighted imaging (DWI) in COVID-19 infections.

Methods: In this retrospective study, Cranial MRI images of 46 adults were evaluated. Group 1 consisted of 27 patients (16 males and 11 females) with positive COVID-19 real-time polymerase chain reaction (RT-PCR) assay. The control group (Group 2) consisted of 27 patients without COVID-19 (16 males, 11 females). In both groups, apparent diffusion coefficient (ADC) values measurements were performed in corpus amygdala, thalamus and insular gyrus.

Results: In COVID 19 positive patients, thoracal CT involvement was detected in $44.4 \%$ and smell loss complaint was present in $40.7 \%$. Lymphopenia was present in $22.2 \%$; and CRP, D-dimer and ferritin values increased. Thalamus ADC values of the group 1 (COVID-19 group) were significantly lower than the control group bilaterally. However insular gyrus and Corpus amygdala ADC values were not different between the COVID-19 and control groups. In each of the groups, $\mathrm{ADC}$ values were not different between the right and left sides. Insular gyrus and corpus amygdala ADC values; and thalamus ADC values showed positive correlations. Insular gyrus ADC values (right) were higher in females. Left insular gyrus and corpus amygdala ADC values were higher in COVID-19 patients with smell loss. Right insular gyrus and left corpus amygdala ADC values were lower in COVID-19 patients with lymphopenia.

Conclusion: Diffusion restriction in olfactory areas can be considered as an obvious indicator of that the immune system is effected and caused damage at neuronal level with the COVID-19 virus, Given the urgency and lethality of the current pandemina, patients with acute onset odor loss should be considered as a high suspicion-adhesive index for the accompanying SARS-CoV-2 infection. CoV infections can affect the nervous system and CoV's host immune mechanisms. These infections can cause permanent damage that can lead to neurological diseases. Therefore, the sense of smell should also be considered and evaluated simultaneously with other neurological symptoms, including headaches, consciousness disorders, paresthesia and other pathological symptoms in patients with $\mathrm{CoV}$ infection. DWI should be widely used as an early imaging method for CNS infections and especially in relation to COVID-19. Treatment of infectious neurological complications is key to improving the prognosis of the critical patients.

1024-TO BE AWARE OF INTRACRANIAL HYPOTENSION; THE RADIOLOGICAL APPROACH

$\underline{\text { Basak Atalay }}{ }^{1}$, Umut Percem Orhan Soylemez ${ }^{1}$

Istanbul Medeniyet University Faculty of Medicine Goztepe City Hospital Radiology Istanbul-Turkey ${ }^{{ }^{I}}$

Purpose: To draw attention to Magnetic resonance imaging (MRI) findings that may spot-on intracranial hypotension (IH). IH is an uncommon, usually self-limiting condition caused by cerebrospinal fluid leakage.

Methods: Sixteen patients diagnosed as IH by cranial MRI and 20 controls were evaluated. Pachmeningeal enhancement, subdural effusions, pituitary hyperplasia, dural sinus distention, tonsillar ectopia, mamillopontine distance, pontomesencephalic angle, and interpeduncular angle were assessed and compared with the control group.

Results: There was no demographic difference between the controls and the patients. Pituitary hyperplasia was seen in all patients. Dural sinus distention was a common finding $(93,7 \%)$, subdural effusion detected in 10 patients $(62,5 \%)$. In all non-contrast MR images, there was pachymeningeal thickness in FLAIR sequences, pachymeningeal enhancement was seen in all MR images with contrast in the patient group. Tonsillar ectopia was the rarest findings (25\%). Mamillopontine distance was significantly lesser in the IH group $(\mathrm{p}=0,002)$. There were no statistically significant differences in pontomesencephalic and interpeduncular angles between the two groups.

Conclusion: Often due to insufficient clinical information, IH is a challenging diagnosis. The awareness of the radiologist is essential in the diagnosis of $\mathrm{IH}$. Comparative evaluation, with previous imaging, may allow early diagnosis with findings such as dural sinus distension and increased pituitary gland size. Pachymeningeal enhancement and subdural fluid collections/hemorrhages may be unremarkable at the beginning; in such cases, dural sinus distention and pituitary gland dimensions become more critical. The dural sinus distension, pituitary gland enlargement, and lesser mamillopontine distance were the most reliable IH diagnosis findings.

\section{6-RESULTS OF CRANIOVERTEBRAL DECOMPRESSION} ON CSF DYNAMICS IN CHIARI I MALFORMATION

Begümhan Baysal ${ }^{1}$, Mahmut Bilal Doğan ${ }^{1}$, Ercan Boşnak ${ }^{2}$, Nejat Ișık ${ }^{2}$ Istanbul Medeniyet University Goztepe Training and Research Hospital Radiology Istanbul-Turkey ${ }^{1}$ Istanbul Medeniyet University Goztepe Training and Research Hospital Neurosurgery Istanbul-Turkey ${ }^{2}$

Purpose:We aimed to compare clinical scoring systems and cerebrospinal fluid (CSF) dynamics obtained by MR imaging in Chiari Malformation Type-1 (CM-1) patients in the preoperative and postoperative period.

Methods: Fifty-five patients diagnosed with CM-1 and 20 healthy volunteers, who underwent CSF MRI between January 2016 and June 2020, were evaluated. 35 of the 55 patients were preoperative and 20 had undergone decompression surgery due to CM-1.

The relationship between preoperative MR findings and clinical findings of 55 patients was evaluated. The changes in clinical and imaging findings of the 20 patients who had undergone surgery were compared.

Results: At the end of cerebellar tonsil level, peak positive and negative velocity (PPV-PNV) of CM-I patients was higher than that of healthy volunteers pre-surgery (PPV:5.43 \pm 3.17 vs $3.91 \pm 1.78 \mathrm{~cm} / \mathrm{s} ; \mathrm{p}=0.006$ ). Net flow of CM-I patients was also lower but not statistically significant $(\mathrm{p}=0.3)$.

While CSF flow was observed homogeneously in the control group, it was nonhomogeneous in the patient group. Aliasing of the reverse flow was seen in 37(67\%) patients.

PPV and PNV were decreased after surgery (PPV:6.23 \pm 2.79 vs $4.81 \pm 1.94 \mathrm{~cm} / \mathrm{s}$, $\mathrm{p}=0.030$; PNV:-7.52 \pm 3.42 vs $-5.10 \pm 1.91, \mathrm{p}=0.014)$. Net Flow was increased ( 0.26 vs $2.15 \mathrm{ml} / \mathrm{min}$; $=0.019$ ).

The postoperative response was worse in patients with more tonsillar herniation, and no significant relationship was found between dynamic flow values and outcome score.

Conclusion:Our findings suggest that the determination of flow dynamics and morphology using MRI findings correlate with clinical findings and may be a useful tool in determining the need and timing of surgery.

1027-DOES DIFFUSION WEIGHTED MAGNETIC RESONANCE IMAGING INFORMATION ABOUT PRIMARY CANCER IN

$\underline{\text { SEVGI YILMAZ SAĞLAM }}{ }^{1}$, FATMA AKTAȘ ${ }^{1}$, ZAFER ÖZMEN ${ }^{1}$ TOGÜ MEDICAL FAKULTY HOSPITAL RADIOLOGY TOKAT-Turkey

Purpose:Metastases in the central nervous system (CNS) significantly affect the prognosis and quality of life of patients with cancer, and approximately $30 \%$ of patients with brain metastases do not have a known 
primary cancer diagnosis at the time of diagnosis. Determining the primary cancer type of these patients as soon as possible is very important in terms of treatment planning and prognosis. The aim of this study is to show whether there is a difference among ADC values depending on the primary focus in patients with metastatic brain tumor, and depending on the type of cancer in patients with primary CNS tumor.

Material and Methods:The study included total 317 patients, 188 of primary and 129 of metastatic, with CNS tumors detected in Diffusion Weighted Magnetic Resonance Imaging (MRI) taken between July 2013 and July 2020 at the TOGU Medical Faculty Hospital. Based on the diffusion weighted images and clinical data of the patients; age, gender, number of lesions, location, and average ADC values were evaluated. Patients with pure cystic or hemorrhagic lesions were excluded from the study. Patients who had undergone radiotherapy or chemotherapy were also excluded from the study, as incorrect results may occur. ADC values were calculated manually regardless of the clinical information of the patients, and of the histological type of the lesions. 3 ROIs were placed in different regions of the lesion and the mean ADC value of these 3 measurements were calculated. Primary masses were divided into 4 groups as low-grade glial mass (13 patients), high grade glial mass (32 patients), meningioma (117 patients), and the others because the number of remaining patients is low (26 patients). Metastatic patients were divided into 3 groups as lung cancer metastasis (77 patients), breast carcinoma metastasis (18) and other metastasis (34) groups.

Results:Mean ADC values of low-stage glial masses were significantly higher than all other groups $(\mathrm{p}<0.001)$. In the metastasis group, the mean $\mathrm{ADC}$ values of the lung metastasis subgroup were significantly higher than the other metastases subgroup ( $p=0.043$ ). In the other metastases subgroup, statistical analysis could not be performed due to the insufficient number of patients, but the mean ADC values of patients with RCC metastases were calculated as approximately $0.521 \times 10-3 \mathrm{~mm}^{2} / \mathrm{sec}$ as lower. In addition, in the lung metastasis subgroup, we found that the ADC values of patients with histopathological diagnosis of SCLC were significantly lower than those of NSCLC. There was no significant difference between advanced stage glial masses and metastases.

Discussion and Conclusion:The mean ADC values of low-grade glial masses were significantly higher than all other groups. However, no significant difference was found between advanced stage glial masses and the other groups. Therefore, we consider that the intratumoral ADC values cannot be used in the differentiation of high grade glial mass from the solitary metastasis. In lung cancer metastases, mean ADC values of SCLC were significantly lower than NSCLC. Therefore, differentiation of SCLC from NSCLC can be made by examining ADC values in patients with brain metastases with a lung tumor whose biopsy cannot be performed. Although statistical analysis could not be performed due to the insufficient number of patients, the mean ADC values of RCC patients in the other metastasis group were found to be $0.521 \times 10-3 \mathrm{~mm}^{2}$ / $\mathrm{sec}$ as lower than other metastases. The ADC values of patients with RCC metastasis may aid diagnosis, but further studies with larger patient groups are needed for more accurate and reliable results. In conclusion, diffusion-weighted conventional MRI makes important contributions in the differential diagnosis of CNS tumors. If supported by studies with large and homogeneous groups, and with the help of other imaging findings, more accurate and reliable results can be reached.

\section{8-CORRELATION OF MORPHOLOGICAL FINDINGS AND} ADC VALUES WITH KI 67 IN GLIOBLASTOM

Süleyman Öncü ${ }^{1}$, İsmail Șerifoğlu ${ }^{1}$

sbü bağcllar suam radyoloji istanbul-Turkey ${ }^{I}$

Purpose: Glioblastoma is the most common primary neoplasm of the central nervous system. Despite improvements in diagnosis and treatment protocols in recent years, its prognosis is quite poor. Rapid progression of the tumor, complete resection generally not possible, and frequent recurrence after treatment are the most common causes of morbidity and mortality. Although the exact diagnosis is made after histopathological examination, it is sometimes inadequate due to insufficient tissue sampling and apparent heterogeneity. The proliferative index of ki-67 is a value that reflects the mitotic index of the tumor found after pathological examination. High ki-67 values are often associated with poor prognosis. In this study, we evaluated the morphological characteristics (peritumoral edema, necrosis, enhacement pattern, heterogeneity, hemorrhage, mass effect, contour irregularity) of the tumor in preoperative MRI in order to contribute histopathological examination noninvasively, predict prognosis and guide treatment protocols. We tried to determine the relationship between the mean values, the total score we obtained from the sum of the scores, and the diffusion MRI parameters (ADC) of tumors with ki-67 values.

Material and methods: 52 patients who were histopathologically diagnosed with glioblastoma and no other known malignancies were included in the study. MRI images of patients taken before surgery and chemoradiotherapy, including postcontrast T1-weighted and diffusion-weighted sequences were retrospectively evaluated. For each tumor, each morphological feature was scored based on its severity or quantity and a total score was obtained. In addition, ADC values of each tumor were measured at the workstation. The ADC value of each tumor and the total score obtained from the morphological findings were compared with the ki-67 values determined as a result of histopathology. In addition, they were divided into groups according to the severity of each morphological feature and the mean ki-67 and ADC values of these groups were compared among themselves.

Results: There was a statistically significant correlation between ki-67 values and ADC values in the negative direction $(r=-0.895 \mathrm{p}=0.0001)$ and in the positive direction $(\mathrm{r}=0.772 \mathrm{p}=0.0001)$ between the total scores. There was a statistically significant negative correlation between the total score and ADC values $(r=-0.780 \mathrm{p}=0.0001)$. Mean ki-67 values of irregular contoured $(\mathrm{p}=0.005)$, midline shifting $(\mathrm{p}=0.005, \mathrm{p}=0.004)$, heterogeneous $(p=0.002, p=0.003)$, significantly enhanced $(p=0.011)$, hemorrhagic $(\mathrm{p}=0.002)$ and necrotic tumors $(\mathrm{p}=0.0001)$ were found to be significantly higher than other groups. There was no statistically significant difference between the mean of ki-67 of the 3 groups divided according to the amount of peritumoral edema $(\mathrm{p}=0.058)$.

Conclusion: It should be kept in mind that the morphological findings and diffusion parameters detected by preoperative MRI are related to ki67 , which can be used to predict early prognosis and guide treatment.

\section{9-COMPARISON OF DISABILITY LEVEL AND MRI FINDINGS IN MULTIPLE SCLEROSIS}

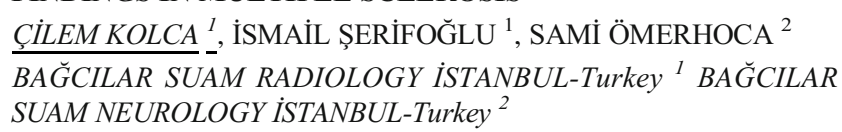

Purpose:Multiple sclerosis is the most common demyelinating disease of the central nervous system. Despite the advances in diagnosis and treatment protocols in recent years, its prognosis is quite variable. Clinical follow-up and repeated imaging are required to predict the course of the disease. EDSS scores are used in clinical follow-up. High EDSS scores are generally associated with poor prognosis. The main modality in radiological follow-up is MRI. In this study, we aimed to determine the relationship between the localization of plaques in follow-up MRI and the development of new plaques with EDSS values in order to predict the prognosis in MS patients.

Material and methods: Sixty-five patients with clinical diagnosis of MS and no other known comorbidities were included in the study. Two consecutive MRI images taken during the routine controls of the patients were evaluated retrospectively. MS plaques were evaluated using conventional MRI sequences (T1WI, T2WI, FLAIR) and T1WI sequences taken after intravenous contrast agent injection. As lesion localizations; frontal, parietal, temporal, occipital lobes, corpus callosum, pericallosal 
area, cerebellar hemispheres, medulla oblangata, pons, brainstem, thalamus, periventricular white matter, subcortical area, posterior fossa, cervical spinal cord, optic nerves, centrum semiovale were used. The examinations were evaluated by a radiologist with 4 years of experience. Clinical EDSS scores were not known during radiological evaluation. EDSS scores were calculated by a neurology specialist trained in scoring. The cases were grouped as "progressed EDSS" with increased EDSS score and "not proggressed EDSS" without any change in EDSS score. In addition, patients with an EDSS score of 2.5 and below were grouped as "early stage" and those with an EDSS score of 3 and above as "advanced stage". EDSS progression status and EDSS score of each case were compared with plaque localization and new plaque development. Cases with contrast enhancement and / or newly developed T2 hyperintense plaque on MRI were grouped as active disease. Disease activity and plaque localization were compared with EDSS scores.

Results: When we evaluate the plaque localizations according to activity; The presence of plaque in the cerebellar hemispheres were significantly in higher frequency in the active patient group $(p=0.026)$. There was no significant relationship between the presence of plaques in other locations and disease activity status.

Proggresed EDSS scores were noted among the patients who developed new plaques in the location of the corpus callosum $(\mathrm{p}=0.011)$. However, no significant relationship was found between the development of new plaques and EDSS progression at other locations.

When we grouped our cases as "early stage" and "advanced stage" according to EDSS, no statistically significant relationship was found between plaque localizations and EDSS level.

Conclusion: Plaque localization and new plaque development detected in MRI are related to the EDSS score and thus, it can be used to predict the prognosis in the early period and direct treatment at follow-up.

\section{8 - E F F I C I E N C Y OF DKI IN EVALUAT ING DEMYELINATING PLAQUES OF MULTIPLE SCLEROSIS}

$\underline{\text { Derya Fidan }}{ }^{1}$, Nail Bulakbaş ${ }^{1}$, Mehmet Alp Dirik ${ }^{1}$, Özgür Tosun ${ }^{2}$

University of Kyrenis, Dr. Suat Gunsel Hospital radiology KyreniaTurkey ${ }^{I}$ Near East University Bioistatistic Nicosia-Turkey ${ }^{2}$

Purpose: Kurtosis is one of the new MR diffusion methods which is reliable in showing microstructural changes in MS patients at an early stage, evaluating demyelination at early stages, and evaluating active demyelinating plaques without using contrast media.

Materials and methods: DKI was acquired with three values of $b(b=0$, $\mathrm{b}=1000$ and $\mathrm{b}=3000 \mathrm{~s} / \mathrm{mm} 2$ ) along 12 diffusion -encoding directions. The DK acquisition time was $2.08 \mathrm{~s}$ with the following sequence parameters; matrix 160x160 mm, FOV $240 \mathrm{~mm}$ and 25 axial slices with a $5 \mathrm{~mm}$ slice thickness and $0.6 \mathrm{~mm}$ gap. Our study included a total of 60 participants. 40 of the participants ( 31 female and 9 male)were between the ages of 19-64 and were diagnosed with Multiple Sclerosis according to the McDonald criteria between 2018-2020. The other 20 participants ( $8 \mathrm{fe}-$ male and 12 male)were included as a control group. We classified active plaques as type 1 , chronic plaques as type 2 , and chronic stable plaques as type 3. The parametric analysis of the diffusion-weighted images of the plaques was performed using the Syngo.via Frontier MR body diffusion 1.0 Siemens MRI program. Here, the average of the diffusion kurtosisand ADC values obtained from the mixed 24 directions were obtained automatically. The obtained datawas evaluated statistically. We compared the control group and each of the MS plaques with the Kruskal Wallstest. If a difference was noted between them, we compared all plaque types with the control group using the Dunn's test.

Results: When comparing the kurtosis and ADC values of the plaques and control group, an increase in signal was noted in all groups compared to the control group.Statistically, the increase in ADC values was the least in type 1 plaques, this was in contrast to the DK values which showed the highest increase in type 1 plaques.
Conclusion: DKI is a reliable advanced diffusion MRI method that can be used in the diagnosis and follow-up of MS disease.

\section{5-DEEP LEARNING FOR AUTOMATED ACUTE ISCHEMIC LESION SEGMENTATION ON DWI}

$\underline{\text { Deniz Alis }}{ }^{1}$, Mert Yergin ${ }^{2}$, Mert Celenk ${ }^{2}$, Omer Bagcilar ${ }^{3}$, Yeseren Senli $^{4}$, Ahmet Ustundag ${ }^{4}$, Vefa Salt ${ }^{4}$, Ceren Alis ${ }^{5}$, Cagdas Topel ${ }^{6}$, Ozan Asmakutlu ${ }^{6}$, Sebahat Dogan ${ }^{7}$, Murat Velioglu ${ }^{8}$, Hakan Selcuk 9', Osman Kizilkilic ${ }^{4}$, Ercan Karaarslan ${ }^{1}$

Acibadem Mehmet Ali Aydinlar University School of Medicine Radiology Istanbul-Turkey ${ }^{I}$ Hevi ai healthcare Artificial Intelligence and information Technologies Istanbul-Turkey ${ }^{2}$ Silivri State Hospital Radiology Istanbul-Turkey ${ }^{3}$ Istanbul University-Cerrahpasa, Cerrahpaşa Medical Faculty Radiology Istanbul-Turkey ${ }^{4}$ Istanbul University-Cerrahpasa, Cerrahpaşa Medical Faculty Neurology Istanbul-Turkey ${ }^{5}$ Istanbul Mehmet Akif Ersoy Thoracic and Cardiovascular Surgery Training and Research Hospital Radiology Istanbul-Turkey ${ }^{6}$ Istanbul Gaziosmanpasa Training and Research Hospital Radiology Istanbul-Turkey ${ }^{7}$ Istanbul Fatih Sultan Mehmet Training and Research Hospital Radiology Istanbul-Turkey ${ }^{8}$ Istanbul Bakirköy Sadi Konuk Training and Research Hospital Radiology Istanbul-Turkey ${ }^{9}$

Purpose: The volume of ischemic core assessed on DWI provides essential insights for decision-making and prognostication. The current gold standard for measuring ischemic core on DWI is manual segmentation, which is a labor-intensive task. In the present work, we used a deep learning architecture, U-net, for automated segmentation of acute ischemic lesions on DWI on a large-scale multi-center data set.

Methods: We reviewed radiology reports of consecutive brain DWI obtained due to clinical suspicion of acute ischemic stroke from five different centers between January 2015 and May 2020. Expert neuroradiologists manually outlined the stroke lesions on high b-value DWI for creating ground-truth. The data set was split into three parts by the ratio of $80 \%, 10 \% 10 \%$, and these parts were used as the training, validation, and test sets, respectively. The primary metric for investigating the model's performance was the Dice coefficient score, which ranges between zero and one where one shows a complete match between ground-truth and a prediction while zero reflects no-match.

Results: A total of 3996 patients, 2544 males, with a mean age of 65.24 years (range, 48-92) were included in the study. The training, validation, and test samples covered 3196, 400, and 400 patients. U-net model yielded Dice coefficient scores of 0.69 and 0.68 for the validation and test tests, respectively. These results were indicated our model yield similar performance compared with that of prior work.

Conclusion: Deep learning-based ischemic lesion segmentation on DWI might provide a valuable contribution to radiologists with good segmentation performance.

\section{0-VISUAL ASSESSMENT OF ASL MRI IN PATIENTS WITH} COGNITIVE PROBLEMS

Murat Dokdok ${ }^{1}$, Meltem H Alpsan Gökmen ${ }^{2}$, Kutlay Karaman ${ }^{1}$, Yasar Kütükçü $^{2}$, Oktay Karadeniz ${ }^{1}$

Anadolu Medical Center Radiology Kocaeli Gebze-Turkey ${ }^{I}$ Anadolu Medical Center Neurology Kocaeli Gebze-Turkey ${ }^{2}$

Purpose: Dementia is an expanding health-care problem that is mostly caused by Alzheimer's disease (AD) in which a pathological process starts years before cognitive impairment gets attention. Arterial Spin Labeling (ASL) magnetic resonance imaging (MRI) offers a non-invasive, and reproducible method for measuring brain perfusion. We aimed to measure consistency of ASL MRI in a heterogenous group of patients with cognitive impairment.

Methods: 85 patients with cognitive problems were included: 23 of the patients had $\mathrm{AD} ; 24$ of the patients had minimal cognitive impairment; 31 
of the patients were diagnosed as having depression and seven of the patients had subjective memory impairment. All patients underwent 3D pulsed ASL MRI where inline calculated CBF maps were evaluated by two neuroradiologists.

Results: Bilateral parietal hypoperfusion patterns were statistically significant in the $\mathrm{AD}$ and MCI groups than depression and SMI groups $(\mathrm{p}<0,001)$. Hypoperfusion rates were \% 67 among MCI and \% 83 in the $\mathrm{AD}$ group. The hypoperfusion rate of ASL maps in depressive patients was $13 \%$ which was significantly lower than $\mathrm{MCI}$ and $\mathrm{AD}$ groups and similar to the SMI group, which did not show any hypoperfusion pattern. After dichotomizing parietal lobe hypoperfusion into present or absent, interobserver agreement evaluated by kappa value ( 0.78 with $95 \%$ $\mathrm{CI}=0.65-0.91)$ indicated good agreement. The complete interobserver agreement was $89 \%$.

Conclusion: ASL MRI could be a valuable method in the evaluation and management of cognitive impairment. Visual assessment used in clinical settings when a quick judgement needed on the presence of perfusion is reproducible.

\section{3-PERFUSION MRI FINDINGS IN PARKINSON'S PATIENTS}

HALIME CEVIK CENKERI I ${ }^{1}$, ATILLA YILMAZ ${ }^{2}$, SADIK AHMET UYANIK $^{1}$, ERAY ATLI ${ }^{1}$, ÜMUT ÖĞÜȘLÜ ${ }^{1}$, BIRNUR YILMAZ ${ }^{1}$, BURÇAK GUMUȘ ${ }^{1}$

OKAN RADIOLOGY ISTANBUL-Turkey ${ }^{1}$ OKAN NEUROSURGEY ISTANBUL-Turkey $^{2}$

Purpose: Parkinson's disease (PD), is a neurodegenerative disease characterized by, the loss of dopaminergic neurons in substantia nigra.

Objective: To contribute to the pathogenesis of the disease by comparing Diffusion tensor (DTI) and contrast perfusion MRI (PWI) findings in medical treatment resistant PD cases.

Material and Methods: Nineteen controls and 21 medical treatmentresistant PD cases, who are candidates for deep brain stimulation, were included in our study. DTI and PWI examination were performed on all cases. Cerebral blood flow (rCBF), mean transit time (MTT) and fractional anisotropy (FA) values were measured in both hemispheres; on primary motor cortex (M1), supplementary motor cortex (SMA), parietal, putamen (P), lateral and medial globus pallidus (GPe / GPi), ventrolateral nucleus (T) of the thalamus, substantia nigra $(\mathrm{SN})$, cuneus, precuneus and cerebellar dentate nucleus (D).

Results: The average age of our control group cases was $59.2 \pm 13.2$ (31$79)$ and the median was 60 years. The average age of PD patient group was $56.7 \pm 16(42-77)$ and the median was 60.5 years. The duration of the disease was $12.4 \pm 7.1$ (5-30) years on average. In PD group, FA values were significantly lower $(p<0.05)$ than the control group, in all regions. In the case group, rCBF-Precuneus on the left, rCBF-SMA, rCBF-Parietal and rCBF-D values on both sides were significantly higher than the control group $(\mathrm{p}<0.05)$. In the case group, MTT-GPi value on the left and MTT-Cuneus value on the right, and MTT-M1, MTT-SMA, MTT-Parietal, MTT-GPe, MTTPrecuneus and MTT-T values on both sides were significantly higher than the control group $(\mathrm{p}<0.05)$.

Conclusion: It is thought that decrease in FA compared to the control group in all regions except the motor cortex may be secondary to cell loss. In PWI, on the other hand, in medical treatment resistant PD cases, rCBF, increases in the supplementary motor and motor cortex, dentate nucleus and left cuneus. MTT, moreover, is prolonged in all regions, evident in left GPi and right cuneus. Perfusion differences may be due to increased blood brain barrier permeability, endothelial degeneration, and abnormal angiogenesis, and may contribute to dopa-resistant symptoms in PD cases.

Keywords: Contrast perfusion, Diffusion tensor (DTI) imaging, MRI, Parkinson.

\section{4-DIFFERENTIATION OF BRAIN METASTASES FROM BRAIN MRI WITH DEEP CNN}

Mehmet Ali Gultekin ${ }^{1}$, Abdusselim Adil Peker ${ }^{1}$, Ayse Betul Oktay ${ }^{2}$, Haci Mehmet Turk ${ }^{3}$, Dilek Hacer Cesme ${ }^{1}$, A $\bar{b}$ dallah T M Shbair ${ }^{3}$, Temel Fatih Yilmaz ${ }^{1}$, Ahmet Kaya ${ }^{1}$, Ayse Irem Yasin ${ }^{3}$, Mesut Seker ${ }^{3}$, Alpaslan Mayadagli ${ }^{4}$, Alpay Alkan ${ }^{1}$

Bezmialem Vakif University Radiology Istanbul-Turkey ${ }^{I}$ Istanbul Medeniyet University Computer Engineering Istanbul-Turkey ${ }^{2}$ Bezmialem Vakif University Medical Oncology Istanbul-Turkey ${ }^{3}$ Bezmialem Vakif University Radiation Oncology Istanbul-Turkey ${ }^{4}$

Purpose: To classify metastatic brain lesions of the lung and breast using convolutional neural network $(\mathrm{CNN})$ in brain magnetic resonance images.

Methods: A total of 143 patients with 157 metastatic brain tumors throughout 2014 to 2019 were enrolled in the study. Manual segmentation of the contrast-enhanced T1-weighted images of the brain has been done prior to radiomics analysis. Three different $\mathrm{CNN}$ architectures that are pre-trained on ImageNet dataset have been employed to differentiate lung and breast metastases. Ten-fold cross-validation used to evaluate the CNNs. Accuracy, precision, recall, F1-score and area under curve (AUC) metrics were calculated to evaluate the performance of the networks.

Results: Xception architecture has the highest accuracy and AUC with 0.83 and 0.79 respectively. VGG19 and the InceptionV3 architectures performed with similar accuracy and AUC metrics (0.8-0.75 and 0.780.74 respectively).

Conclusion: Convolutional neural networks with deep learning features achieved a good diagnostic performance in differentiating brain metastases from lung and breast malignancies. Radiomics analysis of the brain MRIs can be useful in the clinical setting where conventional radiological methods are inadequate and further investigations are required.

\section{0-NEUROIMAGING FINDINGS IN COVID 19 CASES WITH NEUROLOGICAL SYMPTOMS}

$\underline{\text { Buket } Y A \breve{G} C I}{ }^{1}$, İclal ERDEM TOSLAK ${ }^{2}$, Recep BALIK ${ }^{3}$

Haydarpaşa Education and research hospital Radiology Istanbul-Turkey ${ }^{1}$ Antalya Education and research hospital Radiology Antalya-Turkey ${ }^{2}$ Haydarpaşa Education and research hospital Infectious diseases Istanbul-Turkey ${ }^{3}$

Purpose: Coronavirus disease 2019 (COVID-19), which is caused by the highly pathogenic virus severe acute respiratory syndrome coronavirus 2 (SARS-CoV-2), was first detected in Wuhan, China, in December 2019 (1).While respiratory system complications of COVID-19 have been the most common and life-threatening, central and peripheral nervous system involvement has increased. We retrospectively evaluated the Brain CT and MRI examinations of Covid 19 patients with neurological symptoms. In this study, we aimed to show the CNS imaging findings of covid 19 cases and the effect of cerebrovascular damage on the prognosis of the disease.

Methods: Thirty-four patients who were admitted to the emergency department with neurological symptoms and underwent chest imaging and simultaneous CNS imaging were included in the study.

Results: A total of 34 patients, 22(64.7\%) male, 12(35.3\%) female, ranged in age from 29 to 90 . Acute cerebral infarction was detected in 3 patients $(8.8 \%)$, acute-early subacute parenchymal hematoma in one patient(2.9\%), and acute subdural hemorrhage in one patient (2.9\%). In $23(67.6 \%)$ patients, T2 FLAIR hyperintensities on MRI were observed in favor of ischemia. Chronic infarction in 11 $(32.4 \%)$ patients, transverse myelitis in $1(2.9 \%)$ patient, diffuse cerebral edema was detected in 1 patient $(2.9 \%)$. While normal neuroimaging was significantly higher in patients with Covid 
clinical severity 1 , pathological neuroimaging was found significantly higher in patients with clinical severity 3. HT and DM are present in 3 of the patients who develop acute infarction, and acute infarction is significantly more common in patients with HT and DM. It was observed that in patients with comorbid diseases such as HT and DM, Covid 19 pneumonia was more severe and the clinical course was more severe in these patients.

Conclusion: Covid 19 patients with neurological symptoms and pathological neuroimaging findings may have more severe pneumonia and clinical course.

\section{5-CAROTID AND CEREBRAL CTA WITH ULTRA LOW VOLUME CONTRAST MEDIUM}

Mustafa Hizal ${ }^{1}$, Yunus Y1lmazsoy ${ }^{1}$, Süleyman Filiz ${ }^{1}$

Bolu Abant İzet Baysal University Faculty of Medicine Radiology BoluTurkey $^{1}$

Purpose:To evaluate image quality of carotid and cerebral computed tomography angiography (CC CTA) using low amount of iodinated contrast medium $(\mathrm{CM})$ diluted with saline.

Methods:We included a total of 97 patients admitted to our department for CC CTA. In control patients received intravenous administration of $\mathrm{CM}$ at a dose of $45 \mathrm{ml}$ and CTA examinations were performed at $120 \mathrm{kV}$. In ultra low volume(ULV) group, patients received intravenous administration of CM at a dose of $15 \mathrm{ml}$ diuted with $5 \mathrm{ml}$ saline and CTA examinations were performed at $100 \mathrm{kV}$. The same iodinated CM containing $300 \mathrm{mgI} / \mathrm{ml}$ was used in both groups. Attenuation values were measured from specific arterial segments using regions of interest. Subjectie image quality (SIQ) analysis was made using a five point scale. We obtained institutional review board approval.

Results:A total of 56 (35 male, 21 female) and 41 (28 male, 13 female)patients were included in control and ULV groups, respectively. A total of 1940 arterial segments in carotid and vertebrobasilary systems were analyzed. SIQ scores and mean arterial attenuation values was significantly higher in control group (280.56 $\pm 69.32[\mathrm{SD}])$ than ULV group $(374.36 \mathrm{HU} \pm 73.79[\mathrm{SD}])(\mathrm{P}<0.01)$. There was no significant difference between the number of patients with 4 or more SIQ scores, in both groups. There was no significant difference between SIQ scores of the patients with body mass index (BMI) $<25$ in the ULV group and the entire control group ( $\mathrm{p}=0.067$ ).

Conclusion: CC CTA examinations using a 64 dedector CT, could be succesfully obtained, particularly in patients with low BMI,using diluted ULV CM diluted with saline.

\section{8-ADC VALUES IN THE DIFFERENTIATION OF INTRAVENTRICULAR TUMORS}

Bora Korkmazer ${ }^{1}$

Istanbul University-Cerrahpasa Cerrahpasa School of Medicine Radiology Istanbul-Turkey ${ }^{I}$

Purpose: The aim of this study is to investigate the contribution of apparent diffusion coefficient (ADC) values to diagnosis in the differentiation of intraventricular tumors.

Materials and Methods: 48 patients, who were operated for intraventricular mass lesions in Cerrahpasa School of Medicine between 20152020 , were retrosepectively analyzed. Age, gender, lesion location, lesion size, and magnetic resonance imaging findings of the patients were evaluated. Histopathological results of the lesions were compared with the ADC measurement results.

Results: Of the 48 patients operated for intraventricular mass, 26 were female and 22 were male. The mean age of the patients was found to be $31.2 \pm 18.7$ (ranging between 2 and 68 years). As a result of histopathological evaluation, 10 of the lesions were subependymoma (WHO grade
I), 13 of them were central neurocytoma (WHO grade II), 9 of them were subeependimal giant cell tumor (WHO grade I), 9 of them were intraventricular meningioma (WHO grade II), 3 of them were ependymomas (WHO grade II) and 4 of them were diagnosed as anaplastic ependymoma (WHO grade III). A statistically significant difference was found in the comparison of ADC values of intraventricular tumors $(p<0.05)$.

Conclusion: Magnetic resonance imaging findings of intraventricular tumors demonstrate similarities. The use of ADC values in the differentiation of these lesions may contribute to increased diagnostic accuracy.

\section{9-BRAIN MRI VOLUME CHANGES IN SCA DISEASE}

Burcu Düzel Asıg ${ }^{1}$, Gülen Burakgazi ${ }^{2}$, Serdar Doğan ${ }^{3}$, Murat Güntel ${ }^{4}$, Gül Ilhan ${ }^{5}$, Ayça Seyfettin ${ }^{6}$

Hakkari Devlet Hastanesi Radyoloji A.D Radiology Hakkari-Turkey ${ }^{1}$ Recep Tayyip Erdoğan Üniversitesi Tip Fakültesi Radyoloji A.D Radiology Rize-Turkey ${ }^{2}$ Hatay Mustafa Kemal Üniversitesi Tıp Fakültesi Biyokimya A.D Biochemistry Hatay-Turkey ${ }^{3}$ Hatay Mustafa Kemal Üniversitesi Tip Fakültesi Nöroloji A.D Neurology Hatay-Turkey

${ }^{4}$ Hatay Mustafa Kemal Üniversitesi Tip Fakültesi iç Hastalıklarl A.D Internal Medicine Hatay-Turkey ${ }^{5}$ Osmaniye Devlet Hastanesi Radyoloji A.D Radiology Osmaniye-Turkey ${ }^{6}$

Purpose: The main pathophysiology that causes the clinical picture in SCA(sickle cell anemia) is acute vasocclusive events(1,2). Central nervous system is one of the most important systems affected(3). By performing brain MRI(magnetic resonance imaging), manual and automatic volume measurement of the subunits of the brain can be made with the help of various programs and applications. Various serum markers have been evaluated in acute brain ischemia. Some of those are neuronal enzymes such as NSE(Neuron spesific enolase), glial injury and activation markers protein $\mathrm{S} 100 \mathrm{~B}$, periostin and TGF $\beta 1$ (transforming growth factor $\beta 1$ )which increase extracellular matrix production during inflammation, and intermediate filament protein GFAP(glial fibrillary acidic protein) of the central nervous system(4-10).

Our aim in this study is to investigate whether there is a significant change in brain subunit volumes calculated by Volbrain method in patients with sickle cell anemia and to investigate whether there is a relationship between these changes and some biochemical markers (NSE, S100B, Periostin, P-GFAP and TGF- $\beta 1)(11)$.

Materials and methods: Thirty patients who were admitted to the hematology outpatient clinic, who were diagnosed with sickle cell anemia and did not currently have a vasocclusive crisis clinic, and 30 healthy individuals who were matched for age and gender were included in the study. Serum levels of TGF $\beta 1, \mathrm{~S} 100 \mathrm{~B}$, GFAP, Periostin and NSE biomarkers were studied. Non-contrast brain MRI with T1W thin section sequence was performed. Volbrain method was used for brain volume measurements.

Results: There were statistically significant differences between the groups in terms of periostin and NSE values $(p<0.001)$. In volume MRI, a very high level of significant $(p<0.001)$ differences were observed between the groups in white matter, cerebrospinal fluid, total brain volumes and right cerebral hemisphere values. Highly significant differences were found in total cerebral hemispheres $(p=0.001)$ and left cerebral hemisphere $(\mathrm{p}=0.002)$. Bilateral amygdala $(\mathrm{p}=0.012)$, left globus pallidus ( $\mathrm{p}=0.022)$, total globus pallidus $(\mathrm{p}=0.024)$, right hippocampus $(\mathrm{p}=0.025)$, bilateral hippocampus $(\mathrm{p}=0.026)$, left hippocampus $(\mathrm{p}=$ 0.035 ), Significant statistical differences were observed in terms of left amygdala $(p=0.037)$, left nucleus accumbens $(p=0.039)$ and right caudate nucleus $(\mathrm{p}=0.048)$ values.

Discussion and Conclusion: In sickle cell anemia, brain volume calculation by Volbrain method, NSE and Periostin measurement were investigated for the first time in the literature in our study, and significant differences were found between patient and control groups. Although similar results were obtained with studies using conventional MRI in SAD patients in the 
literature, the findings were evaluated as quantitative data in our study (1215). Brain volume changes that could not be detected by conventional MRI were determined quantitatively in our study. There was no significant correlation between Periostin and NSE levels and volume MRI findings.

1074-DO SIGMOID\& JUGULAR NOTCH AREAS PREDICT THE INTRACRANIAL HEMORRHAGE SIDE

$\underline{\text { Elif Dilara Topcuoglu }}{ }^{1}$, Yasar Bukte ${ }^{1}$

Umraniye Education and Research Hospital Radiology Istanbul-Turkey ${ }^{1}$

Purpose: Intraparenchymal cerebral hemorrhage (ICH) is a lifethreatening condition and results from a non-traumatic arteriole rupture and bleeding into the brain parenchyma. Jugular and sigmoid notches are the gates for brain venous outflow. In the current study, we aimed to evaluate the association between the side of ICH and the sigmoid and jugular notch areas.

Materials and Methods: In this retrospective study, neuroradiology case records were evaluated to identify patients with $\mathrm{ICH}$ who underwent cranial CT examination. Patients with normal unenhanced cranial CTs were also assessed. Jugular and sigmoid notch areas were calculated bilaterally for both groups and compared by student $t$ test.

Results: A total of 265 patients (111 women and 154 men) with a mean age of $68.8 \pm 11.7$ years (range between 50 to 90 years), were enrolled. Control group harbored 45 patients (19 women and 26 men) with a mean age of $68.3 \pm 11.9$ years (range between 50 to 90 years). Right and left sided ICHs were detected in 140 and 125 patients, respectively. No significant difference was found in terms of right jugular and right sigmoid notch areas between patients with right sided ICH and control group $(\mathrm{P}=0.483$ and $\mathrm{P}=0.156$, respectively). No significant difference was found in terms of left jugular and left sigmoid notch areas between patients with left sided ICH and control group $(\mathrm{P}=0.449$ and $\mathrm{P}=0.118$, respectively).

Conclusion: Sigmoid and jugular notch areas were not significantly associated with the side of ICH and did not predict the side of $\mathrm{ICH}$, in this series.

\section{7-COMPARISON OF KI 67 PROLIFERATION INDEX VALUES OF MENINGIOMAS WITH ADC}

$\underline{\text { Bülent Yıldız }}{ }^{1}$, Ertan Ünver ${ }^{1}$

Sivas Cumhuriyet University School of Medicine Radiology Sivas-Turkey

Purpose: The aim of this study was to investigate the efficacy of DWI findings in predicting the histological degree of meningiomas preoperatively and the relationship between ADC value and the most important prognosis determinant index, Ki-67 proliferation index..

Methods: Between March 2013 - July 2020, 54 patients who underwent cranial MRI including diffusion, who had a pathological outcome as meningioma, and whose $\mathrm{Ki}-67$ index was examined, were analyzed retrospectively. The threshold ADC value for low and high grade tumor separation was calculated by ROC analysis. The correlation of Ki-67 proliferation index and $\mathrm{ADC}$ values was investigated.

Results: The mean ADC value in low-grade meningiomas was found as $976,674 \pm 105,443 \times 10-6 \mathrm{~mm} 2 / \mathrm{sec}$, and the average ADC value in high-grade meningiomas was $813,727 \pm 102,998 \times 10-6 \mathrm{~mm} 2 /$ $\mathrm{sec}$ and this difference was found statistically significant $(\mathrm{p}<$ 0,001). The threshold ADC value was found to be $882.50 \times 10-6$ $\mathrm{mm} 2 / \mathrm{s}(\mathrm{p}<0.05)$, sensitivity at this value was calculated as $83.7 \%$ and specificity as $81.8 \%$. The Ki-67 proliferation index was $5.77 \pm$ 2.438 in low-grade meningiomas and $16 \pm 8.955$ in high-grade tumors, and these differences were statistically significant $(\mathrm{p}<0.001)$. There was a significant negative correlation (correlation) between Ki-67 index and mean ADC values $(p=0.008)$.
Conclusion: DWI could be used in the differentiated between low and high grade meningiomas.Ki-67 proliferation index and average ADC values can be used together in terms of meningioma grade.

1087-BLOOD PRESSURE LIMITS AFFECTING CAROTID ARTERY INJURY.

Aylin Güneșli ${ }^{1}$, Çiğdem Yalçın ${ }^{1}$

Baskent University Radiology Adana-Turkey ${ }^{l}$

Purpose: Although it is well known that high blood pressure causes undesirable effects on carotid arteries, it is not clear as to which threshold value this effect starts. The aim of this study is to evaluate and determine this threshold.

Materials and Methods: This cross-sectional study included a total of 308 individuals in the following groups; Group 1: individuals with systolic blood pressure (SBP) $<120 \mathrm{mmHg}$ and diastolic blood pressure (DBP) $<80 \mathrm{mmHg}$ (optimal), Group 2: SBP 120-129 mmHg and/or DBP 80-84 mmHg (normal), Group 3: SBP 130-139 mmHg and/or DBP 85-89 mmHg (high-normal), Group 4: SBP 140-159 mmHg and/ or DBP 90-99 mmHg, Group 5: SBP 160-179 $\mathrm{mmHg}$ and/or DBP 100$109 \mathrm{mmHg}$, and Group 6: $\mathrm{SBP} \geq 180 \mathrm{mmHg}$ and/or $\mathrm{DBP} \geq 110 \mathrm{mmHg}$. Carotid distensibility and elasticity were measured in all groups.

Results: A statistically significant difference was determined between the groups in carotid distensibility and elasticity ( $\mathrm{p}<0.001$, for both values). There was no significant difference between groups 2 and 1 ( $p>0.05$ ), but there was a significant difference between other groups (groups 3-4-5) and group 1 ( $\mathrm{p}<0.05$, for all comparisons). SBP $>135.5 \mathrm{mmHg}$ started to affect carotid distensibility with $78.2 \%$ sensitivity and $74.6 \%$ specificity, and DBP $>86.5 \mathrm{mmHg}$ with $79.3 \%$ sensitivity and $71.6 \%$ specificity. Carotid elasticity was seen to be affected by SBP $>137.5 \mathrm{mmHg}$ with $80.4 \%$ sensitivity and $73.1 \%$ specificity, and $\mathrm{DBP}>88.5 \mathrm{mmHg}$ with $79.1 \%$ sensitivity and $73.8 \%$ specificity.

Conclusion: Carotid distensibility and elasticity decreases with increasing blood pressure. In individuals with high-normal blood pressure, carotid damage may begin after a certain blood pressure value.

\section{0-CRANIAL MAGNETIC RESONANCE IMAGINGN HYPOTONIC INFANTS WITH COBALAMIN DEFICIENCY}

$\underline{\text { Beril Dilber }}{ }^{1}$, İlker Eyüboğlu ${ }^{2}$

karadeniz technichal university pediatric neurology TRABZON-Turkey ${ }^{1}$ karadeniz, technichal university radiology TRABZON-Turkey ${ }^{2}$

Purpose: Vitamin B12 begins to accumulate in infants within the first six months while mothers often remain asymptomatic and infantile vitamin B12 deficiency may not be noticed until the onset of neurological effects. In infants with $\mathrm{Cbl}$ deficiency, long-term exposure to elevated methylmalonic acid and homocysteine (MMA-HC) may have toxic effects on the central nervous system.

Method: The aim of this study was to evaluate cranial magnetic resonance (MRI) findings of 22 hypotonic infants that were followed up with a diagnosis of nutritional $\mathrm{Cbl}$ deficiency and combined MMA-HC.

Results: Of the 78 infants that presented with hypotonicity, 22 (28.2\%) infants were detected with vitamin B12 deficiency. Elevated MMA-HC levels were detected in all patients $(100 \%)$. Cranial MRI showed cortical atrophy in $6(28.5 \%)$-large sylvian fissures in 7 (33.3\%)-ventricular dilatation in $5(23.8 \%)$-corpus callosal thinning in $6(28.5 \%)$-delayed myelination in $2(10 \%)$, and normal in $8(36.3 \%)$ infants. Infants detected with corpus callosal thinning and cortical atrophy on MRI had significantly lower vitamin B12 levels $(\mathrm{p}<0.05)$.

Conclusion: Vitamin B12 deficiency is a treatable condition, it should be suspected in infants presenting with hypotonicity, neuro-developmental delay. Neuroradiological findings should be considered in the diagnosis of such patients. İnfantile nutritional vitamin B12 deficiency, which can 
be a source of numerous persistent neurological deficits and mental retardation during the long term, should be treated to allow the patient to allow healthy neuro-development for infants. Maternal and fetal vitamin B12 levels should be assessed during the third trimester of pregnancy to prevent long-term exposure to infantile vitamin B12 deficiency.

\section{0-REASONS FOR ADMISSION TO HOSPITAL OF CHILDREN WITH OCCIPITAL PETALIA}

Merter KEÇELI ${ }^{1}$, Miraç Yıldırım ${ }^{2}$

Konya Sehir Hastanesi Pediatric Radiology Konya-Turkey ${ }^{I}$ Ankara University Medical Faculty Pediatric Neurology Ankara- ${ }^{2}$

Purpose: Occipital petalia (OP) is an anatomical definition in which one of the occipital lobes is bent to the opposite side. In this study, the relationship between the reasons for admission to hospital in patients under 15 years of age and OP detected in cranial magnetic resonance imaging (MRI) was investigated.

Methods: Sixty-five children who applied to the pediatric neurology outpatient clinic between January 2017 and January 2020 and did not have any findings other than OP in MRI were included in the study. Occipital bending direction was determined according to the displacement of the fissure between the occipital lobes with respect to the midline on MRI. Children with a history of cranial operation and trauma, children with known and significant anomalies, patients with metabolic disease, myelinization pathology, organ failure and syndromic children were excluded from the study. Information on hand preference, clinical findings and patient complaints were also collected from all participants. The side where the occipital lobes were angled was determined.

Results: The mean age of the patients was $8 \pm 1.25 .45$ of the cases were male and 20 were female. While $50(77 \%)$ of the patients had right bending, $15(23 \%)$ had left bending. The presence or direction of bending was not associated with hand dominance $(\mathrm{p}>0.12)$. There was no correlation between gender and direction of OP $(p>0.20)$. Forty-eight percent of the patients were imaged for nonspecific headache, $21 \%$ for epilepsy, $8 \%$ for visual impairment, $17 \%$ for fainting-loss of consciousness etiology, $6 \%$ for determining possible complications of hypertension and congenital heart diseases. There was no child diagnosed with a psychiatric illness. Cavum septum pellicidum was detected in 12 cases (18\%). TS dominance was observed on the opposite side of the direction where OP was detected in MRI.

Discussion: The most common reason for admission to the hospital in patients with OP in the pediatric age group is headache. Studies to be carried out in larger series in cases with association of OP and anomalyradiopathology may provide supportive information on this issue. The OP bending direction is related to the TS hypoplasia aspect. In this respect, $\mathrm{OP}$ can be used as a diagnostic clue in dural venous evaluation.

\section{4-ROLE OF MRI INTHE DIAGNOSIS OF FETALCENTRAL NERVOUS SYSTEM ANOMALIES}

Ismail MESE ${ }^{1}$, Bahattin OZKUL ${ }^{2}$, Ceylan ALTINTAS TASLICAY ${ }^{3}$, Yasemin DOGAN ${ }^{4}$, Yonca ANIK ${ }^{3}$

Erenkoy Mental Health and Neurological Disease Training and Research Hospital Radiology Istanbul-Turkey ${ }^{1}$ Istanbul Bilim University, Sişli Florence Nightingale Hospital Radiology IstanbulTurkey ${ }^{2}$ Kocaeli University Radiology Kocaeli-Turkey ${ }^{3}$ Kocaeli University Obstetrics and Gynecology Kocaeli-Turkey ${ }^{4}$

Purpose: To assess the role of MRI in the diagnosis of fetal central nervous system (CNS) anomalies and to investigate whether the technique improves the accuracy of US for the relevant evaluation.

Materials and methods:207 consecutive patients, between October 2014 and March 2019, were referred to fetal MRI and were included in our prospective study after detection or suspicion of fetal anomalies by US. 55 patients could not be assessed postnatally since they did not come to our hospital for follow-up. 81 cases had CNS anomalies and 63 cases had the other system anomalies. Also 24 cases were found normal. Gestational age range was 16-38 weeks and maternal age range was 1844 years. US was performed all patients by obstetrician 1-2 week ago before fetal MRI.152 new borns were assessed for confirming prenatal diagnosis by any radiological modality(CT, MRI and US) or autopsy.

Results: In 54 patients US and MRI were revealed same CNS anomalies but additional anomalies having been missed by US were detected in 16 patients. Furthermore diagnosis was changed in 2 patients based on MRI findings and in 6 patients fetal anomalies were found by MRI despite US revealed no abnormal finding. Neverthless, in 3 cases various fetal anomalies were found by US instead of MRI. According to the results MRI provided more information in 24 patients compared to US.

Conclusions: Fetal MRI, having a complementary role to US, is an important tool for the diagnosis of fetal CNS anomalies and can be used for defining the anomaly in details and detecting additional anomalies.

\section{7-EVALUATION OF BASAL GANGLIA BY DTI IN TUBEROUS SCLEROSIS}

DILEK HACER CESME ${ }^{1}$

Bezmialem Vakif University Radiology Istanbul-Turkey ${ }^{\text {I }}$

Purpose: Self-injurious behaviors are chronic and repetitive selfdirected behavioral actions that are physically damaging and can lead to tissue damage. It is observed in $10-41 \%$ of patients with Tuberous sclerosis (TSC). The aim of our study is to investigate whether there is any difference ADC and FA values obtained from basal ganglia between patients with TSC and healthy control group.

Methods: Twenty five patients with TSC and 15 healthy controls were included the study. Routine MRI and DTI applied to all subjects. Apparent diffusion coefficient (ADC), fractional anisotropy (FA) values were measured from globus pallidum, caudate nucleus and putamen. $\mathrm{ADC}$ and FA values obtained from TSC and healthy control groups were compared.

Results: Increased ADC values obtained from globus pallidum, caudate nucleus and putamen were detected in patients with TSC compared to healthy controls. There was no statistical difference in terms of FA values.

Conclusions: We are speculated that the development of microstructural damage in the basal ganglia of patients with TSC. Increased $\mathrm{ADC}$ values in the basal ganglia may cause a decrease in the number of axons and an increase in extracellular fluid due to damage to the myelin sheath. More studies based on a larger population should be done to better define how microstructural damage in the basal ganglia causes self-injurious behaviors dysfunctions in TSC.

\section{3-OPTIC NERVE EVALUATION IN NF I TIPS FOR OPTIC GLIOMA}

Seda Kaynak Şahap $^{1}$, Sonay Aydın ${ }^{2}$, Betül Emine Derinkuyu ${ }^{1}$

Dr. Sami Ulus Children Hospital Pediatric Radiology Ankara-Turkey ${ }^{1}$ Erzincan University Medicine Faculty Radiology Erzincan-Turkey ${ }^{2}$

Purpose: Neurofibromatosis type I (NF1) is a multisystemic genetic disease in which optic nerve gliomas (ONG) develops frequently. Despite the ONGs are WHO grade I tumors, they may cause progressive vision loss, so early diagnosis is mandatory. The purpose of this study is to evaluate the optic nerve thickness and the presence of tortuosity in patients with NF1 with or without ONG and control subjects and to be able to make more objective criteria for the diagnosis of ONG.

Materials And Methods:MR images of fourty-one patients with NF1 (82 optic nerves) and age-gender matched 41 control subjects (82 optic nerves) were evaluated retrospectively. A pediatric radiologist evaluated the optic nerve thickness at multiple locations. Also the 
presence of the tortuosity and the subarachnoid dilatation was reported. Kruskal-Wallis and Bonferroni post-hoc analysis was used.

Results: The mean diameter of the optic nerve was statistically significant $(\mathrm{p}<0.05)$ in patients with NF1 with OSG compared with those with NF1 without OSG and with control subjects at all locations except chiasmatic segments. Also tortuosity was more common in the NF1 with OSG compared with NF1 without OSG and control group (\%70, \%38 and \%25.7, respectively).

Conclusion: The knowledge of normal values of optic nerves in pediatric patients and effective use of optic nerve thickness and tortuosity in the diagnosis of ONG may reduce the use of contrast agents and related side effects.

\section{4-CHILDHOOD ADRENOLEUKODYSTROPHY MRI FINDINGS \\ $\underline{\text { Berna Uçan }}{ }^{1}$, Betül Emine Derinkuyu ${ }^{1}$ \\ Dr. Sami Ulus Children Hospital Pediatric Radiology Ankara-Turkey ${ }^{l}$}

Purpose:X-linked adrenoleukodystrophy (X-ALD) is a metabolic disorder emerging as a result of the accumulation of very long chain fatty acids in several tissues including the brain. Magnetic resonance imaging (MRI) is the main imaging modality for the characterization and localization of the white matter involvement. We report on the MRI findings of cerebral X-ALD in 12 pediatric patient that followed-up in our center.

Patients and methods:Data of 12 patients diagnosed with ALD were included. Demographic data, age on set initial symptoms, MRI findings were documented. The findings of the conventional MR sequences with intravenous contrast agents were noted. For the analysis of MRI, we used the Loes scoring system.

Results:Data of 12 patients were retrospectively evaluated. Mean age of initiation of symptoms were 15 , male/female ratio was $12 / 1$. On Brain MRI, patient's Loes scores varied between 0-13. The scores of 4 patientare $<4,4$ patients are between 4 and 8 , and 4 patients are between 9 and 13. The most common Loes pattern was 1 . Among the patients, 4 had corticospinal tract lesions without splenium or genu involvement, 6 had symmetric corticospinal tract lesions with additional involvement of the splenium or genu, and 3 did not have corticospinal tract involvement but had other white matter lesions.

Conclusion:Cerebral involvement of patients with X-ALD range widely. Neuroimaging studies suggest that MRI patterns of demyelination in cerebral X-ALD. Therefore, contrast-enhanced MRI is the principal imaging modality for the diagnosis and treatment follow-up of ALD.

1121-DOES GADOPENTETATE DIMEGLUMINE INDUCE GADOLINIUM ACCUMULATION IN THE BRAINS OF CHILDREN

$\underline{\text { Aysegul Gormez }}{ }_{-}$, Osman Melih Topcuoglu ${ }^{1}$

Yeditepe University Radiology Istanbul-Turkey ${ }^{1}$

Purpose: To determine T1-weighted (T1W) signal intensity (SI) differences in the dentate nucleus (DN) and globus pallidus (GP) following contrast enhanced magnetic resonance imaging (MRI) with multiple gadopentetate dimeglumine administrations in a group of pediatric patients.

Methods and materials: This retrospective study included children with at least 2 enhanced brain MRIs. All patients received gadopentetate dimeglumine intravenously $(0.1 \mathrm{mmol} / \mathrm{kg})$. SI measurements were done by drawing 5 region of interests (ROI) on dentate nuclei (DN) and globus pallidi (GP) bilaterally and cerebro-spinal fluid (CSF) in unenhanced T1W images. Student t- test was used for comparison of SI. Pearson correlation was calculated for the correlation between the SI and the number of gadolinium administrations.

Results: A total of 31 children (age range: $3-17$ years; mean $10.6 \pm 4.3$ years) were included. The mean number of administrations was 3.9 \pm 2.1 . There was no significant difference between the first and the last MRI scans: right and left $\mathrm{DN} / \mathrm{CSF}$, right and left $\mathrm{GP} / \mathrm{CSF}(\mathrm{P}=0.543, \mathrm{P}=0.599$, $\mathrm{P}=0.803, \mathrm{P}=0.730$, respectively). Correlation between the number of gadopentetate dimeglumine administrations and the SI for right and left $\mathrm{DN} / \mathrm{CSF}$, right and left GP/CSF $(\mathrm{r}=0.13, \mathrm{r}=0.13, \mathrm{r}=0.09$ and $\mathrm{r}=0.12$, respectively) was poor.

Conclusion: There was no significant T1 SI increase for children with at least 2 gadopentetate dimeglumine administrations in this series.

\section{0-DIFFERENTIATION OF BENIGN CHANGES FROM PROGRESSION IN THE SURGICAL CAVITY}

Ekim Gumeler ${ }^{1}$, Gokcen Coban Cifci ${ }^{1}$

Hacettepe University Radiology Ankara-Turkey ${ }^{I}$

Introduction: Imaging follow-up of patients with brain tumors who had surgery has challenges in regards to the differentiation of residual/ recurrent tumor from treatment-related changes. As the survival of these patients has been increasing, the differentiation of treatment-related changes has gained significant importance.

Material and Method: Here we described four patients who demonstrated a slowly growing nodular enhancement in/around the surgical cavity on their follow-up with advanced magnetic resonance imaging (MRI) such as susceptibility-weighted imaging (SWI), diffusion-weighted imaging (DWI), and dynamic susceptibility contrast perfusion-weighted imaging (DSC-PWI).

Results: Patient 1 with pilocytic astrocytoma and patient 2 with pleomorphic xanthoastrocytoma underwent only total surgical resection, patient 3 with medulloblastoma completed chemoradiation following the total surgical resection. Patient 4 with lymphoma had an excisional biopsy and had chemo-therapy afterward. All patients demonstrated a T2 hypointense nodular lesion in the surgical cavity, which slowly increased in size. The SWI demonstrated a significant signal loss. Also, the enhancement of the lesion after contrast material injection was slowly progressive during follow-up. However, DWI and DSC-PWI showed no signs of recurrence. Patients 1 and 2 showed a cortical vein in association with the lesion.

Conclusion: Progressively enhancing nodular lesion mimicking recurrency can be differentiated from recurrence with advanced MRI, however, RANO criteria still not recommend advanced MRI during follow-up of the post-operative brain tumors.

1042-OTOSCLEROSIS THE DIMENSIONS OF THE COCHLEAR AQUEDUCT AND THE VESTIBULAR AQUEDUCT

$\underline{\text { Vefa Cakmak }}^{1}$, Pinar Cakmak $^{1}$

Pamukkale University Radiology denizli-Turkey ${ }^{1}$

Objective: The aim of this study is to investigate the relationship between cochlear Aquaduct(CA) and vestibular Aquaduct(VA) dimentions in otosclerosis and otosclerosis types.

Materials and methods: Between January 2018 and September 2020, the temporal bone high resolution Computed Tomography (HRCT) images of 88 patients (46 men, 42 women; range, 20-71 age; mean age, $43,89 \pm 11.66$ years) consisting of 44 otosclerosis patients and 44 control groups were retrospectively evaluated in the radiology archive of our hospital. HRCT examination was performed on 128 detector and 16 detector multi-slice scanners. CA width, CA funnel base width, CA funnel length were measured in axial HRCT images in all patients. CA length 
was positioned on the coronal reformatted image and measured while the round window and the CA funnel base were in the same plane. VA length and width were measured on axial HRCT images. In statistical analysis, the independent sample $t$ test was performed to compare CA and VA dimensions with the control group.

Results: In patients with otosclerosis, there was a significant decrease in both side CA width and right CA funnel width compared to the control group $(\mathrm{p}<0.0001)$. There was no significant difference in VA dimensions, CA length, CA funnel lengths and left CA funnel width in otosclerosis patients compared to the control group. There was no statistically significant difference in CA and VA dimensions for the fenestral and retrofenestral types.

Conclusion: The vestibular aquaduct due to an increase in bone tissue in the otic capsule and antefenestrum is unaffected, while the cochlear aquaduct narrows.

Publisher's note Springer Nature remains neutral with regard to jurisdictional claims in published maps and institutional affiliations. 